\title{
Critical behavior of weakly interacting bosons: A functional renormalization group approach
}

\author{
Nils Hasselmann, Sascha Ledowski, and Peter Kopietz \\ Institut für Theoretische Physik, Universität Frankfurt, \\ Robert-Mayer-Strasse 8, 60054 Frankfurt, Germany
}

(Dated: November 5, 2018)

\begin{abstract}
We present a detailed investigation of the momentum-dependent self-energy $\Sigma(k)$ at zero frequency of weakly interacting bosons at the critical temperature $T_{c}$ of Bose-Einstein condensation in dimensions $3 \leq D<4$. Applying the functional renormalization group, we calculate the universal scaling function for the self-energy at zero frequency but at all wave vectors within an approximation which truncates the flow equations of the irreducible vertices at the four-point level. The self-energy interpolates between the critical regime $k \ll k_{c}$ and the short-wavelength regime $k \gg k_{c}$, where $k_{c}$ is the crossover scale. In the critical regime, the self-energy correctly approaches the asymptotic behavior $\Sigma(k) \propto k^{2-\eta}$, and in the short-wavelength regime the behavior is $\Sigma(k) \propto k^{2(D-3)}$ in $D>3$. In $D=3$, we recover the logarithmic divergence $\Sigma(k) \propto \ln \left(k / k_{c}\right)$ encountered in perturbation theory. Our approach yields the crossover scale $k_{c}$ as well as a reasonable estimate for the critical exponent $\eta$ in $D=3$. From our scaling function we find for the interaction-induced shift in $T_{c}$ in three dimensions, $\Delta T_{c} / T_{c}=1.23 a n^{1 / 3}$, where $a$ is the s-wave scattering length and $n$ is the density, in excellent agreement with other approaches. We also discuss the flow of marginal parameters in $D=3$ and extend our truncation scheme of the renormalization group equations by including the six- and eight-point vertex, which yields an improved estimate for the anomalous dimension $\eta \approx 0.0513$. We further calculate the constant $\lim _{k \rightarrow 0} \Sigma(k) / k^{2-\eta}$ and find good agreement with recent Monte-Carlo data.
\end{abstract}

PACS numbers: 03.75.Hh, 05.30.Jp, 05.70.Jk

\section{INTRODUCTION}

The physics of weakly interacting bosons has seen a revival thanks to the improved experimental technique of atom traps which allow for a detailed study of BoseEinstein condensation in a controlled environment. Besides the theoretical effort invested into studies of harmonically trapped bosons (for a review, see Ref. 11]), this also generated renewed interest into the behavior of homogeneous Bose gases and led to some new insights [2]). Many aspects of weakly interacting Bose gases have been understood for quite some time now, such as the universality class $O(2)$ of the condensation transition along with an accurate knowledge of critical exponents. However, the universality of weakly interacting bosons at the critical point is not limited to critical exponents since a weakly interacting Bose gas behaves universal at all length scales larger than the thermal de-Broglie wavelength $\lambda_{\mathrm{th}}$ [3, 4]. The origin of this extended universality is that at large length scales quantum fluctuations become unimportant so that this regime of the Bose gas is completely described by a classical $\phi^{4}$-model 5]. In this work we present a detailed study of the momentumdependence of the self-energy at the critical point of BoseEinstein condensation at zero frequency, using the functional renormalization group formalism in the form introduced by Wetterich [6] and by Morris [7]. Some results of this manuscript were already presented in a brief form [8]. Here we give a detailed account of the calculation and further include an extensive treatment of marginal terms.

In the limit of weak interactions, parametrized by the s-wave scattering length $a$, the self-energy is universal not only in the limit of small wave vectors, $\mathbf{k} \rightarrow 0$, where it is proportional to $k^{2-\eta}$ with a finite anomalous dimension $\eta$. It remains universal, in the sense that it can be written in a scaling form independent of $a$, up to momenta which only need to be small compared to $\lambda_{\text {th }}^{-1}$. At some crossover scale $k_{c}$ the zero frequency self-energy leaves the anomalous scaling regime and enters the perturbative regime, where the self-energy correction to the dispersion becomes negligible compared with the bare dispersion

$$
\epsilon_{\mathbf{k}}=\rho_{0} \mathbf{k}^{2}, \quad \text { with } \rho_{0}=\hbar^{2} / 2 m \text {. }
$$

Here $m$ is the bare mass. At finite temperatures and $D<4$, a perturbative calculation of the self-energy is ultraviolet (UV) divergent. This divergence can be addressed by using the thermal de-Broglie length as an UV cutoff. In $D=3$, however, the perturbative regime $k_{c}<k<2 \pi / \lambda_{\text {th }}$ remains non-trivial even in presence of a UV cutoff, since additional logarithmic infra-red (IR) divergences appear. As discussed in detail by Baym et al. 5], the IR divergence cannot be treated in an ad-hoc manner by introducing an IR cutoff, since doing so introduces an artificial additional scale which directly enters quantities which should be universal, such as the interaction induced shift of the critical temperature. The IR divergence can be removed by a re-summation using a variety of standard many-body techniques, e. g. bubbleor ladder-summation and/or self-consistent approaches [5]. However, these methods are uncontrolled in the critical regime where one is faced with a strong-coupling problem. Renormalization group $(\mathrm{RG})$ techniques are expected to perform better and several authors have ap- 
plied RG techniques to investigate the IR behavior of weakly interacting bosons 9, 10, 11], though no attempt was made to calculate the momentum dependence of the self-energy. Note that standard field theoretical RG is confined to the critical regime $k \ll k_{c}$. In fact, even the scale $k_{c}$ cannot be obtained within such an approach. To interpolate between the critical and the short wavelength regime, functional $\mathrm{RG}$ techniques $[6,7]$ are a natural choice, since they track the flow of complete vertex functions rather than just a small number of coupling parameters. Note that the functional RG approach includes a priori also terms which are irrelevant according to their scaling behavior, but which are important for a correct description of the perturbative $k_{c} \ll k$ regime. In this work we present in detail a functional RG approach to this problem and calculate the universal self-energy to leading order in the small parameter $a n^{1 / 3}$ for all wave vectors ( $n$ is the boson density). Since the critical behavior at $T_{c}$ is classical, we will focus only on the self-energy $\Sigma\left(\mathbf{k}, i \omega_{n}=0\right)$ at vanishing Matsubara frequency.

We begin with the general form of the functional RG flow equations for the irreducible vertices up to the fourpoint vertex in Sec. III where we still retain all frequencies. Our approach is based on the sharp-cutoff version of the functional RG [6, 7, 12, 13. In Sec. III] we turn to the effective classical field theory and rewrite the problem in a notation appropriate to the classical limit. In Sec. IIIA the relevant and marginal parameters are classified in dimensions $3<D<4$ and their functional flow equations are stated in Sec. IIIB

Sec. IV contains the central part of our work, the calculation of the self-energy. We first re-examine perturbation theory and its divergence in $D=3$ before we turn to the calculation of the self-energy within the functional RG formalism. This formalism allows us to calculate the scaling function $\sigma(x)$ characterizing the zero frequency self-energy at the critical point. We define $\sigma(x)$ by

$$
\sigma(x)=\left(\rho_{0} k_{c}^{2}\right)^{-1}\left[\Sigma\left(k_{c} x\right)-\Sigma(0)\right],
$$

such that the crossover occurs at $x=1$. Here $\Sigma(k)=$ $\Sigma\left(k, i \omega_{n}=0\right)$ is the exact zero frequency self-energy at the critical temperature. Standard field theoretical RG can only describe the asymptotic limit $x \rightarrow 0$, where $\sigma(x) \propto x^{2-\eta}$.

Our approach is based on a truncation of the exact hierarchy of functional RG flow equations at the four-point vertex, i. e. we ignore six-point and higher order vertices. While in this approximation the flow of marginal terms is not consistently described, the resulting approximation for the four-point vertex does in fact include marginal and infinitely many irrelevant terms. The resulting flow equation for the self-energy can then be solved and we derive the complete momentum dependence for the selfenergy in $3 \leq D<4$, see Eqs. 4.51] 4.52) below, which constitute the central result of this work. A numerical evaluation of Eqs. 4.51 4.52) in $D=3$ is also presented. We use $\sigma(x)$ to calculate the shift of the critical temperature $T_{c}$ of the condensation transition in Sec. $\mathbf{D}$ In
$D=3$ we obtain $\Delta T_{c} / T_{c}=1.23 a n^{1 / 3}$ to lowest order in $a n^{1 / 3}$, in good agreement with recent numerical investigations [14, 15] and other analytical results [16, 17] (for a recent review on this topic see [2]).

In Sec. VI we improve upon the truncation of the flow equation and account for the coupling parameters which become marginal in $D=3$. Three additional parameters must be taken into account, two of which are associated with the linear momentum dependence of the fourpoint vertex and one which describes the momentumindependent part of the six-point vertex. We discuss different truncation schemes of the flow equations and show that the inclusion of marginal terms provide an improvement for the fixed point value of the anomalous dimension.

In Sec. VII we calculate $\sigma(x)$ in $D=3$ including the marginal terms of the four-point vertex but ignoring irrelevant terms. We demonstrate how this truncation fails in the large $x$ regime, where it predicts incorrectly $\sigma(x) \propto x$ which in turn would predict $\Delta T_{c} / T_{c} \propto a n^{1 / 3} \ln \left(a n^{1 / 3}\right)$. On the other hand, in the critical regime irrelevant terms only lead to a renormalization of marginal and relevant ones and $\sigma(x)$ is well described by a theory were irrelevant terms are not included. We use this approach to express the prefactor $A_{3}$ of the anomalous scaling term, $\sigma(x) \approx A_{3} x^{2-\eta}$, as a function of $\eta$ for $D=3$. Our result is in good agreement with recent Monte Carlo results [4]. Finally, in Sec. VIII we summarize and conclude this work.

\section{FUNCTIONAL RG FLOW EQUATIONS FOR BOSONS}

Our starting point is a standard effective action describing free bosons with a two-particle interaction, which is local at the bare level. The action with an UV cutoff $\Lambda_{0}$ (to be specified later) is of the form

$$
S_{\Lambda_{0}}\{\bar{\psi}, \psi\}=S_{\Lambda_{0}}^{0}\{\bar{\psi}, \psi\}+S_{\Lambda_{0}}^{\mathrm{int}}\{\bar{\psi}, \psi\},
$$

where the non-interacting part is given by

$$
\begin{aligned}
& S_{\Lambda_{0}}^{0}\{\bar{\psi}, \psi\}=\int_{K} \Theta\left(\Lambda_{0}-|\mathbf{k}|\right) \\
& \times\left[-i \omega_{n}+\epsilon_{\mathbf{k}}-\mu+\Sigma(0, i 0)\right] \bar{\psi}_{K} \psi_{K},
\end{aligned}
$$

with $\Theta(x>0)=1$ and $\Theta(x<0)=0$. The interaction part is given by

$$
\begin{aligned}
& S_{\Lambda_{0}}^{\mathrm{int}}\{\bar{\psi}, \psi\}= \\
& \quad \int_{K} \Theta\left(\Lambda_{0}-|\mathbf{k}|\right)\left[\Sigma_{\Lambda_{0}}(K)-\Sigma(0, i 0)\right] \bar{\psi}_{K} \psi_{K} \\
& \quad+\frac{1}{(2 !)^{2}} \int_{K_{1}^{\prime}} \int_{K_{2}^{\prime}} \int_{K_{2}} \int_{K_{1}} \delta_{K_{1}^{\prime}+K_{2}^{\prime}, K_{2}+K_{1}} \\
& \quad \times \Gamma_{\Lambda_{0}}^{(4)}\left(K_{1}^{\prime}, K_{2}^{\prime} ; K_{2}, K_{1}\right) \bar{\psi}_{K_{1}^{\prime}} \bar{\psi}_{K_{2}^{\prime}} \psi_{K_{2}} \psi_{K_{1}}+\ldots,
\end{aligned}
$$

where the ellipsis denotes three-body and higher order interactions, which we ignore at the bare level. Here $\psi_{K}$ is a 
complex bosonic field. We use the notation $K=\left(\mathbf{k}, i \omega_{n}\right)$, $\int_{K}=(\beta V)^{-1} \sum_{\mathbf{k}, \omega_{n}}$, and $\delta_{K K^{\prime}}=\beta V \delta_{\mathbf{k k}^{\prime}} \delta_{\omega_{n} \omega_{n^{\prime}}}$, where $\beta$ is the inverse temperature, $V$ is the volume, and $\omega_{n}=$ $2 \pi n T$ are bosonic Matsubara frequencies. In Eq. (2.2) we have included the exact self-energy at vanishing momenta and frequencies as a counterterm in the definition of the free action. Throughout this work, we shall work at temperatures $T \geq T_{c}$ such that the $U(1)$ symmetry is not broken. The generating functional of the one-particle irreducible $n$-point vertices $\Gamma_{\Lambda}^{(2 n)}\left(K_{1}^{\prime}, \ldots, K_{n}^{\prime}, K_{n}, \ldots, K_{1}\right)$ of the theory with IR cutoff $\Lambda$ can be expanded in terms of the fields $\phi_{K}=\left\langle\psi_{K}\right\rangle$ as follows

$$
\begin{aligned}
\Gamma_{\Lambda}\{\bar{\phi}, \phi\} & =\sum_{n=0}^{\infty} \frac{1}{(n !)^{2}} \prod_{i, j=1}^{n} \int_{K_{i}^{\prime}} \int_{K_{j}} \delta_{K_{1}^{\prime}+\ldots+K_{n}^{\prime}, K_{n}+\ldots+K_{1}} \\
& \times \Gamma_{\Lambda}^{(2 n)}\left(\left\{K_{i}^{\prime}, K_{j}\right\}\right) \bar{\phi}_{K_{1}^{\prime}} \cdots \bar{\phi}_{K_{n}^{\prime}} \phi_{K_{n}} \cdots \phi_{K_{1}} \cdot(2.4)
\end{aligned}
$$

The functional RG flow equations of the first few irreducible vertices (up to the six-point vertex) for nonrelativistic fermionic and bosonic many-body systems can be found in Ref. 13. We summarize below the flow equations relevant for bosons up to the four-point vertex. The flow equations for the six-point vertex and some terms of the flow of the eight-point vertex are given in Appendix A

\section{A. Free energy}

For completeness, we list here the flow equation for the free energy $\Gamma_{\Lambda}^{(0)}$, although below we shall not discuss it further. The flow equation is given by

$$
\begin{aligned}
\partial_{\Lambda} \Gamma_{\Lambda}^{(0)} & =V \int_{K} \delta(\Lambda-|\mathbf{k}|) \\
& \times \ln \left[\frac{i \omega_{n}-\epsilon_{\mathbf{k}}+\mu-\Sigma(0, i 0)}{i \omega_{n}-\epsilon_{\mathbf{k}}+\mu-\Sigma(0, i 0)-\Gamma_{\Lambda}^{(2)}(K)}\right],
\end{aligned}
$$

where $\Gamma_{\Lambda}^{(2)}(K)$ is the two-point vertex, defined by

$$
\Gamma_{\Lambda}^{(2)}(K)=\Sigma_{\Lambda}(K)-\Sigma(0, i 0)
$$

\section{B. Two-point vertex}

The main interest of the work is the flow equation of the two point vertex, which we will relate in Sec.[IV to the zero energy scaling function of the self-energy, Eq. (1.2). The flow equation for the two-point vertex is given by

$$
\partial_{\Lambda} \Gamma_{\Lambda}^{(2)}(K)=\int_{K^{\prime}} \dot{G}_{\Lambda}\left(K^{\prime}\right) \Gamma_{\Lambda}^{(4)}\left(K, K^{\prime} ; K^{\prime}, K\right)
$$

where

$$
\dot{G}_{\Lambda}(K)=\frac{\delta(\Lambda-|\mathbf{k}|)}{i \omega_{n}-\epsilon_{\mathbf{k}}+\mu-\Sigma(0, i 0)-\Gamma_{\Lambda}^{(2)}(K)}
$$

is the cutoff dependent single scale propagator, with support only at $|\mathbf{k}|=\Lambda$. As is evident from Eq. (2.7), the flow of the two point vertex depends on the properties of the four-point vertex at finite wave vectors and we thus need to derive the momentum-dependent flow of the fourpoint vertex.

\section{Four-point vertex}

The flow equation for the irreducible four-point vertex is responsible for the cross-over from the weak coupling regime at small scales to the critical regime at large scales. The flow is given by

$$
\begin{aligned}
& \partial_{\Lambda} \Gamma_{\Lambda}^{(4)}\left(K_{1}^{\prime}, K_{2}^{\prime} ; K_{2}, K_{1}\right)=\int_{K} \dot{G}_{\Lambda}(K) \Gamma_{\Lambda}^{(6)}\left(K_{1}^{\prime}, K_{2}^{\prime}, K ; K, K_{2}, K_{1}\right) \\
& \quad+\int_{K}\left[\dot{G}_{\Lambda}(K) G_{\Lambda}\left(K^{\prime}\right) \Gamma_{\Lambda}^{(4)}\left(K_{1}^{\prime}, K_{2}^{\prime} ; K^{\prime}, K\right) \Gamma_{\Lambda}^{(4)}\left(K, K^{\prime}, K_{2}, K_{1}\right)\right]_{K^{\prime}=K_{1}+K_{2}-K} \\
& \quad+\int_{K}\left[\left[\dot{G}_{\Lambda}(K) G_{\Lambda}\left(K^{\prime}\right)+G_{\Lambda}(K) \dot{G}_{\Lambda}\left(K^{\prime}\right)\right] \Gamma_{\Lambda}^{(4)}\left(K_{1}^{\prime}, K^{\prime} ; K, K_{1}\right) \Gamma_{\Lambda}^{(4)}\left(K_{2}^{\prime}, K ; K^{\prime}, K_{2}\right)\right]_{K^{\prime}=K_{1}-K_{1}^{\prime}+K} \\
& \quad+\int_{K}\left[\left[\dot{G}_{\Lambda}(K) G_{\Lambda}\left(K^{\prime}\right)+G_{\Lambda}(K) \dot{G}_{\Lambda}\left(K^{\prime}\right)\right] \Gamma_{\Lambda}^{(4)}\left(K_{2}^{\prime}, K^{\prime} ; K, K_{1}\right) \Gamma_{\Lambda}^{(4)}\left(K_{1}^{\prime}, K ; K^{\prime}, K_{2}\right)\right]_{K^{\prime}=K_{1}-K_{2}^{\prime}+K}
\end{aligned}
$$

where $\Gamma_{\Lambda}^{(6)}$ is the six-point vertex and

$$
G_{\Lambda}(K)=\frac{\Theta\left(\Lambda<|\mathbf{k}|<\Lambda_{0}\right)}{i \omega_{n}-\epsilon_{\mathbf{k}}+\mu-\Sigma(0, i 0)-\Gamma_{\Lambda}^{(2)}(K)}
$$

is the cutoff regularised propagator. The notation $\Theta(\Lambda<$ $\left.|\mathbf{k}|<\Lambda_{0}\right)$ is shorthand for $\Theta(|\mathbf{k}|-\Lambda)-\Theta\left(|\mathbf{k}|-\Lambda_{0}\right)$. This equation is shown graphically in Fig. 11 For the most part of this work, we shall in fact ignore the contribution 


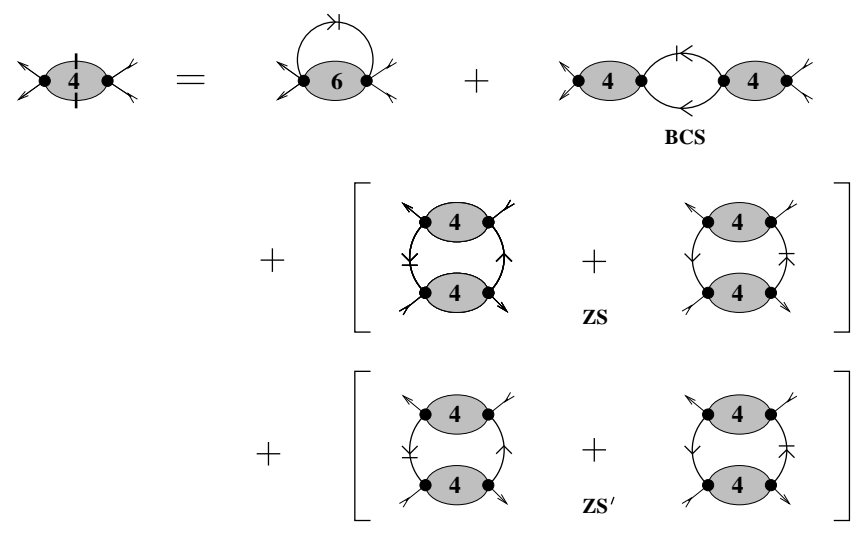

FIG. 1: Diagrammatic representation of the flow equation for the four-point vertex, see Eq. (2.9). We adapt the notation BCS, ZS and $\mathrm{ZS}^{\prime}$ for the diagrams from the usual fermionic language [18], even though this notation does not imply a physical correspondence.

from the six-point vertex to this flow and work solely with the two- and four-point vertex. To calculate fixedpoint properties, we will however also include the sixpoint vertex in Sec. VI

\section{EFFECTIVE CLASSICAL FIELD THEORY}

To discuss the classical critical behavior, it is sufficient to retain only the zero Matsubara frequency part of all vertices. In principle, all fields with non-zero Matsubara frequencies can be integrated out using the flow equations given above, which leads to finite renormalizations of the parameters appearing in the effective classical theory [19]. The effective UV cutoff of the classical theory is determined by the thermal de Broglie wavelength,

$$
\Lambda_{0}=\frac{2 \pi}{\lambda_{\mathrm{th}}}, \quad \lambda_{\mathrm{th}}=\frac{h}{\sqrt{2 \pi m T}}=\sqrt{\frac{2 \pi \hbar^{2}}{m T}} .
$$

In $D=3$ the initial value of the four-point vertex can be parameterized in terms of the two-body scattering length $a$,

$$
\Gamma_{\Lambda_{0}}^{(4)}(0,0 ; 0,0)=\frac{8 \pi \hbar^{2} a}{m}=16 \pi \rho_{0} a
$$

\section{A. Functional RG flow equations for the rescaled vertices}

We assume that the finite renormalizations due to nonzero Matsubara frequencies are implicitly taken into account via the initial conditions at scale $\Lambda=\Lambda_{0}$ of the effective classical theory. As discussed in detail in Ref. 5], to calculate the linear shift of the critical temperature (see Sec. $\nabla$ ), one can in fact ignore the renormalization of the classical sector by finite Matsubara frequencies altogether, since this only leads to corrections in $T_{c}$ of order $a^{2}$. It is convenient to write the single-particle Green's function at zero Matsubara frequency in the following scaling form

$$
\begin{gathered}
G_{\Lambda}(\mathbf{k}, i 0)=-\frac{Z_{l}}{\rho_{0} \Lambda^{2}} \tilde{G}_{l}(\mathbf{k} \Lambda) \\
\tilde{G}_{l}(\mathbf{q})=\frac{\Theta\left(1<|\mathbf{q}|<e^{l}\right)}{R_{l}(\mathbf{q})},
\end{gathered}
$$

were the minus sign is introduced in Eq. (3.3) to arrive at the usual definition of the classical Green's function. Here $l=-\ln \left(\Lambda / \Lambda_{0}\right)$ is the logarithmic flow parameter, and the inverse dimensionless propagator is

$$
R_{l}(\mathbf{q})=Z_{l} \mathbf{q}^{2}+\tilde{\Gamma}_{l}^{(2)}(\mathbf{q})+\frac{Z_{l}}{\rho_{0} \Lambda^{2}}[\Sigma(0, i 0)-\mu],
$$

with the dimensionless irreducible two-point vertex

$$
\begin{aligned}
\tilde{\Gamma}_{l}^{(2)}(\mathbf{q}) & =\frac{Z_{l}}{\rho_{0} \Lambda^{2}} \Gamma_{\Lambda}^{(2)}(\Lambda \mathbf{q}, i 0) \\
& =\frac{Z_{l}}{\rho_{0} \Lambda^{2}}\left[\Sigma_{\Lambda}(\Lambda \mathbf{q}, i 0)-\Sigma(0, i 0)\right]
\end{aligned}
$$

The classical wave-function normalization factor $Z_{l}$ is given by

$$
Z_{l}=1-\left.\frac{\partial \tilde{\Gamma}_{l}^{(2)}(\mathbf{q})}{\partial q^{2}}\right|_{q^{2}=0}
$$

Similar to Eq. (3.6), we define the classical dimensionless higher order vertices for $n \geq 2$ by

$$
\begin{aligned}
& \tilde{\Gamma}_{l}^{(2 n)}\left(\mathbf{q}_{1}^{\prime}, \ldots, \mathbf{q}_{n}^{\prime} ; \mathbf{q}_{n}, \ldots, \mathbf{q}_{1}\right)=\left(K_{D} T\right)^{n-1} \\
& \quad \times \Lambda^{D(n-1)-2 n}\left(Z_{l} / \rho_{0}\right)^{n} \Gamma_{\Lambda}^{(2 n)}\left(\left\{\mathbf{k}_{i}=\Lambda \mathbf{q}_{i}, \omega_{n_{i}}=0\right\}\right) .
\end{aligned}
$$

For later convenience we have included the numerical factor $K_{D}=\Omega_{D} /(2 \pi)^{D}$ in the definition of the vertices, where $\Omega_{D}=2 \pi^{D / 2} / \Gamma(D / 2)$ is the surface area of the $D$-dimensional unit sphere. The rescaled vertices satisfy functional flow equations of the form

$$
\begin{aligned}
& \partial_{l} \tilde{\Gamma}_{l}^{(2 n)}\left(\left\{\mathbf{q}_{i}\right\}\right)= \\
& {\left[2 n-D(n-1)-n \eta_{l}-\sum_{i=1}^{2 n} \mathbf{q}_{i} \cdot \nabla_{\mathbf{q}_{i}}\right] \tilde{\Gamma}_{l}^{(2 n)}\left(\left\{\mathbf{q}_{i}\right\}\right)} \\
& +\dot{\Gamma}_{l}^{(2 n)}\left(\left\{\mathbf{q}_{i}\right\}\right),
\end{aligned}
$$

where

$$
\eta_{l}=-\partial_{l} \ln Z_{l}
$$

is the flowing anomalous dimension. In particular, the rescaled two-point vertex satisfies

$$
\partial_{l} \tilde{\Gamma}_{l}^{(2)}(\mathbf{q})=\left[2-\eta_{l}-\mathbf{q} \cdot \nabla_{\mathbf{q}}\right] \tilde{\Gamma}_{l}^{(2)}(\mathbf{q})+\dot{\Gamma}_{l}^{(2)}(\mathbf{q})
$$


where

$$
\dot{\Gamma}_{l}^{(2)}(\mathbf{q})=\int_{\mathbf{q}^{\prime}} \dot{G}_{l}\left(\mathbf{q}^{\prime}\right) \tilde{\Gamma}_{l}^{(4)}\left(\mathbf{q}, \mathbf{q}^{\prime} ; \mathbf{q}^{\prime}, \mathbf{q}\right)
$$

with

$$
\int_{\mathbf{q}^{\prime}}=\int \frac{d^{D} q^{\prime}}{\Omega_{D}}
$$

and

$$
\dot{G}_{l}(\mathbf{q})=\frac{\delta(|\mathbf{q}|-1)}{R_{l}(\mathbf{q})}
$$

The functional flow equation for the dimensionless fourpoint vertex follows from Eq. (3.9). It involves the inhomogeneity

$$
\begin{aligned}
\dot{\Gamma}_{l}^{(4)}\left(\mathbf{q}_{1}^{\prime}, \mathbf{q}_{2}^{\prime} ; \mathbf{q}_{2}, \mathbf{q}_{1}\right)= & \int_{\mathbf{q}} \dot{G}_{l}(\mathbf{q}) \tilde{\Gamma}_{l}^{(6)}\left(\mathbf{q}_{1}^{\prime}, \mathbf{q}_{2}^{\prime}, \mathbf{q} ; \mathbf{q}, \mathbf{q}_{2}, \mathbf{q}_{1}\right)-\int_{\mathbf{q}}\left[\dot{G}_{l}(\mathbf{q}) \tilde{G}_{l}\left(\mathbf{q}^{\prime}\right) \tilde{\Gamma}_{l}^{(4)}\left(\mathbf{q}_{1}^{\prime}, \mathbf{q}_{2}^{\prime} ; \mathbf{q}^{\prime}, \mathbf{q}\right) \tilde{\Gamma}_{l}^{(4)}\left(\mathbf{q}, \mathbf{q}^{\prime} ; \mathbf{q}_{2}, \mathbf{q}_{1}\right)\right]_{\mathbf{q}^{\prime}=\mathbf{q}_{1}+\mathbf{q}_{2}-\mathbf{q}} \\
& -\int_{\mathbf{q}}\left[\left[\dot{G}_{l}(\mathbf{q}) \tilde{G}_{l}\left(\mathbf{q}^{\prime}\right)+\tilde{G}_{l}(\mathbf{q}) \dot{G}_{l}\left(\mathbf{q}^{\prime}\right)\right] \tilde{\Gamma}_{l}^{(4)}\left(\mathbf{q}_{1}^{\prime}, \mathbf{q}^{\prime} ; \mathbf{q}, \mathbf{q}_{1}\right) \tilde{\Gamma}_{l}^{(4)}\left(\mathbf{q}_{2}^{\prime}, \mathbf{q} ; \mathbf{q}^{\prime}, \mathbf{q}_{2}\right)\right]_{\mathbf{q}^{\prime}=\mathbf{q}_{1}-\mathbf{q}_{1}^{\prime}+\mathbf{q}} \\
& -\int_{\mathbf{q}}\left[\left[\dot{G}_{l}(\mathbf{q}) \tilde{G}_{l}\left(\mathbf{q}^{\prime}\right)+\tilde{G}_{l}(\mathbf{q}) \dot{G}_{l}\left(\mathbf{q}^{\prime}\right)\right] \tilde{\Gamma}_{l}^{(4)}\left(\mathbf{q}_{2}^{\prime}, \mathbf{q}^{\prime} ; \mathbf{q}, \mathbf{q}_{1}\right) \tilde{\Gamma}_{l}^{(4)}\left(\mathbf{q}_{1}^{\prime}, \mathbf{q} ; \mathbf{q}^{\prime}, \mathbf{q}_{2}\right)\right]_{\mathbf{q}^{\prime}=\mathbf{q}_{1}-\mathbf{q}_{2}^{\prime}+\mathbf{q}}
\end{aligned}
$$

The flow equations for the six-point and eight-point vertices also have the form Eq. (3.9) where $\dot{\Gamma}_{l}^{(6)}$ and $\dot{\Gamma}_{l}^{(8)}$ involve various combinations of the two-, six-, four-, eightand ten-point vertices. All terms entering the inhomogeneity of the six-point vertex and the terms needed for a calculation up to second order in the relevant and marginal parameters of the inhomogeneity of the eightpoint can be found in Appendix see Eqs. (A2 A3 .

\section{B. Classification of coupling parameters}

Although we are ultimately interested in the flow of vertex functions, it is useful to first consider the flow of marginal and relevant coupling parameters, since irrelevant parameters become local functions of the relevant and marginal ones at the fixed point [20]. To properly organize the flow of irrelevant terms it is thus necessary to know the flow of the relevant and marginal parameters. We first investigate here the relevant and marginal terms for $3<D<4$, ignoring additional marginal terms in $D=3$.

\section{Relevant coupling parameters}

In $3 \leq D<4$ there are two relevant coupling parameters,

$$
r_{l}=\tilde{\Gamma}_{l}^{(2)}(0)=\lim _{\mathbf{q} \rightarrow 0} \frac{Z_{l}}{\rho_{0} \Lambda^{2}}\left[\Sigma_{\Lambda}(\Lambda \mathbf{q}, i 0)-\Sigma(0, i 0)\right]
$$

with scaling dimension +2 , and

$$
u_{l}=\tilde{\Gamma}_{l}^{(4)}(0,0 ; 0,0)
$$

with scaling dimension $\epsilon=4-D$. The exact flow equations of these parameters are

$$
\begin{gathered}
\partial_{l} r_{l}=\left(2-\eta_{l}\right) r_{l}+\dot{\Gamma}_{l}^{(2)}(0), \\
\partial_{l} u_{l}=\left(4-D-2 \eta_{l}\right) u_{l}+\dot{\Gamma}_{l}^{(4)}(0,0 ; 0,0) .
\end{gathered}
$$

\section{Marginal coupling parameter}

For $3<D<4$ the only marginal parameter is the wave-function renormalization $Z_{l}$. The exact flow equation is

$$
\partial_{l} Z_{l}=-\eta_{l} Z_{l}
$$

where the flowing anomalous dimension is

$$
\begin{aligned}
\eta_{l} & =\left.\frac{\partial \dot{\Gamma}_{l}^{(2)}(\mathbf{q})}{\partial q^{2}}\right|_{q^{2}=0} \\
& =\left.\frac{1}{R_{l}(1)} \frac{\partial}{\partial q^{2}}\left\langle\tilde{\Gamma}_{l}^{(4)}\left(\mathbf{q}, \hat{\mathbf{q}}^{\prime} ; \hat{\mathbf{q}}^{\prime}, \mathbf{q}\right)\right\rangle_{\hat{\mathbf{q}}^{\prime}}\right|_{q^{2}=0}
\end{aligned}
$$

and

$$
\langle\ldots\rangle_{\hat{\mathbf{q}}}=\int \frac{d^{D} \mathbf{q}}{\Omega_{D}} \delta(|\mathbf{q}|-1) \ldots
$$

denotes the integral over the unit sphere. The surface area $\Omega_{D}$ of the unit sphere was defined below Eq. (3.8). In $D=3$, additional marginal coupling parameters appear which we ignore in this section. We will discuss them in Sec. VI 


\section{The Wilson-Fisher fixed point close to $D=4$}

It is instructive to take a closer look at the perturbative one-loop RG flow equations for the relevant coupling parameters. If we retain from the two-point vertex only the relevant and marginal part, we may approximate

$$
R_{l}(\mathbf{q}) \approx r_{l}+\mathbf{q}^{2},
$$

so that

$$
\begin{gathered}
\tilde{G}_{l}(\mathbf{q}) \approx \frac{\Theta\left(1<|\mathbf{q}|<e^{l}\right)}{r_{l}+\mathbf{q}^{2}}, \\
\dot{G}_{l}(\mathbf{q}) \approx \frac{\delta(|\mathbf{q}|-1)}{r_{l}+\mathbf{q}^{2}} .
\end{gathered}
$$

If in addition we retain only the relevant part of the four-point vertex, we may replace it by a momentumindependent constant,

$$
\tilde{\Gamma}_{l}^{(4)}\left(\mathbf{q}_{1}^{\prime}, \mathbf{q}_{2}^{\prime} ; \mathbf{q}_{2}, \mathbf{q}_{1}\right) \approx \tilde{\Gamma}_{l}^{(4)}(0,0 ; 0,0) \equiv u_{l} .
$$

Within this approximation we obtain from Eq. (3.12),

$$
\dot{\Gamma}_{l}^{(2)}(0) \approx \frac{u_{l}}{1+r_{l}},
$$

and Eq. (3.15) reduces to

$$
\dot{\Gamma}_{l}^{(4)}(0,0 ; 0,0) \approx-\frac{5}{2} \frac{u_{l}^{2}}{\left(1+r_{l}\right)^{2}} .
$$

Because the anomalous dimension is related to the momentum dependence of the four-point vertex which is irrelevant in $D>3$, we may set $\eta_{l} \approx 0$ within this approximation. Furthermore, on the critical trajectory $r_{l}=O\left(u_{l}\right)$ can also be ignored in Eq. 3.28) as long as $u_{l}$ remains small compared with unity. Then we obtain, with $\epsilon=4-D$,

$$
\begin{gathered}
\partial_{l} u_{l}=\epsilon u_{l}-\frac{5}{2} u_{l}^{2}, \\
\partial_{l} r_{l}=2 r_{l}+u_{l} .
\end{gathered}
$$

Eq. (3.29) is easily solved. The solution can be written in the form

$$
\frac{u_{l}}{u_{*}}=1-\frac{1}{e^{\epsilon\left(l-l_{c}\right)}+1}=\frac{1}{e^{\epsilon\left(l_{c}-l\right)}+1},
$$

where

$$
u_{*}=\frac{2}{5} \epsilon
$$

is the value of $u_{l}$ at the RG fixed point, and the logarithmic crossover scale is

$$
l_{c}=\frac{1}{\epsilon} \ln \left(\frac{u_{*}}{u_{0}}-1\right) \approx \frac{1}{\epsilon} \ln \left(\frac{u_{*}}{u_{0}}\right) .
$$

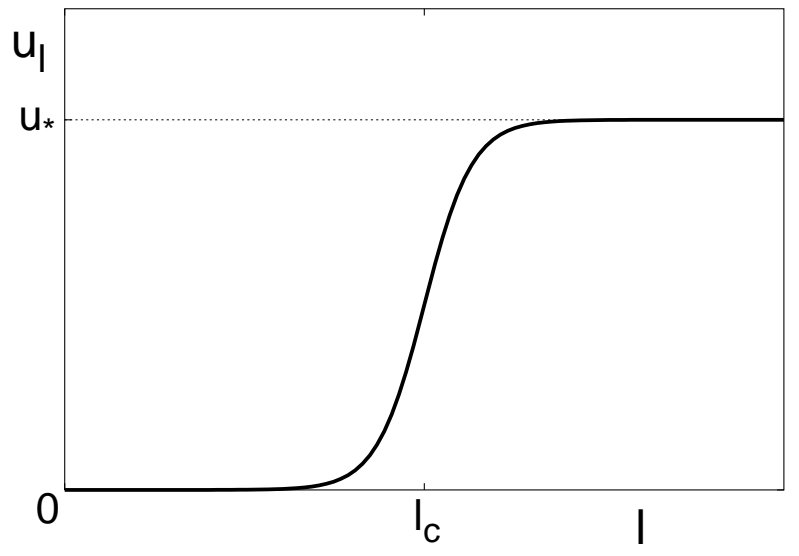

FIG. 2: Typical flow of the coupling $u_{l}$ as a function of $l$ for $u_{0} \ll u_{*}$ and $D=3$.

Throughout this work we assume that $u_{0} \ll u_{*}$, corresponding to weak bare interactions between the bosons. Note that the right-hand side of Eq. (3.31) is expressed in terms of the Fermi function. If we think of $\epsilon$ as the inverse temperature and $l_{c}$ as the chemical potential, the qualitative behavior of $u_{l} / u_{*}$ is clear: within a narrow (on the scale $l_{c}$ ) interval of width $1 / \epsilon$ centered at $l=l_{c}$ the ratio $u_{l} / u_{*}$ raises from the small value $e^{-\epsilon\left(l_{c}-l\right)}$ to a value close to unity, see Fig. 22 Given the solution $u_{l}$ in Eq. (3.31), the RG equation (3.30) for $r_{l}$ is easily solved,

$$
r_{l}=-\frac{\epsilon}{5}+e^{2 l}\left[r_{0}+\frac{\epsilon}{5}-\frac{2 \epsilon}{5} \int_{0}^{l} d l^{\prime} \frac{e^{-2 l^{\prime}}}{e^{\epsilon\left(l^{\prime}-l_{c}\right)}+1}\right] .
$$

To obtain a fixed point at $l \rightarrow \infty$, the intial value $r_{0}$ has to be fine tuned such that

$$
r_{0}=-\frac{\epsilon}{5}+\frac{2 \epsilon}{5} \int_{0}^{\infty} d l^{\prime} \frac{e^{-2 l^{\prime}}}{e^{\epsilon\left(l^{\prime}-l_{c}\right)}+1} .
$$

Then we may write

$$
r_{l}=r_{*}+u_{*} \int_{l}^{\infty} d l^{\prime} \frac{e^{-2\left(l^{\prime}-l\right)}}{e^{\epsilon\left(l^{\prime}-l_{c}\right)}+1},
$$

where

$$
r_{*}=-\frac{u_{*}}{2}=-\frac{\epsilon}{5} .
$$

In Sec. IV we show that, up to a numerical factor of the order of unity, the momentum scale $k_{c}=\Lambda_{0} e^{-l_{c}}$ associated with the logarithmic scale factor $l_{c}$, i. e.

$$
k_{c}=\Lambda_{0}\left[\frac{u_{*}}{u_{0}}-1\right]^{-1 / \epsilon} \approx \Lambda_{0}\left(\frac{u_{0}}{u_{*}}\right)^{1 / \epsilon}
$$

can be identified with the crossover scale where the critical $k^{2-\eta}$-form of the energy dispersion begins to emerge. 


\section{THE SELF-ENERGY AT THE CRITICAL POINT OF BOSE-EINSTEIN CONDENSATION}

\section{A. Second order perturbation theory}

To begin with, let us attempt to calculate the selfenergy by means of straightforward second order perturbation theory [5], which yields in a continuum model with UV cutoff $\Lambda_{0}$

$$
\begin{aligned}
\Sigma(\mathbf{k})-\Sigma(0)= & -T \frac{3}{2} \Gamma_{0}^{2} \int \frac{d^{D} p}{(2 \pi)^{D}} \chi_{0}(\mathbf{p}) \\
& \times\left[\frac{\Theta\left(\Lambda_{0}-|\mathbf{p}+\mathbf{k}|\right)}{\epsilon_{\mathbf{p}+\mathbf{k}}}-\frac{\Theta\left(\Lambda_{0}-|\mathbf{p}|\right)}{\epsilon_{\mathbf{p}}}\right],
\end{aligned}
$$

where $\Gamma_{0}=\Gamma_{\Lambda_{0}}^{(4)}(0,0 ; 0,0)$ is the bare vertex, and

$$
\chi_{0}(\mathbf{p})=T \int \frac{d^{D} p^{\prime}}{(2 \pi)^{D}} \frac{\Theta\left(\Lambda_{0}-\left|\mathbf{p}^{\prime}\right|\right) \Theta\left(\Lambda_{0}-\left|\mathbf{p}^{\prime}+\mathbf{p}\right|\right)}{\epsilon_{\mathbf{p}^{\prime}} \epsilon_{\mathbf{p}^{\prime}+\mathbf{p}}}
$$

With $\epsilon_{\mathbf{p}}=\rho_{0} \mathbf{p}^{2}$ we obtain for $\Lambda_{0} \rightarrow \infty$,

$$
\chi_{0}(\mathbf{p})=K_{D} K_{D}^{\prime} \frac{T}{\rho_{0}^{2} p^{4-D}}
$$

where

$$
\begin{aligned}
K_{D}^{\prime} & =\int_{0}^{1} d x[x(1-x)]^{(D-4) / 2} \int_{0}^{\infty} d y \frac{y^{D-1}}{\left(y^{2}+1\right)^{2}} \\
& =2^{3-D} \frac{\sqrt{\pi} \Gamma\left(\frac{D}{2}-1\right)}{\Gamma\left(\frac{D-1}{2}\right)} \frac{\frac{\pi}{4}(D-2)}{\sin \left(\frac{\pi}{2}(D-2)\right.} .
\end{aligned}
$$

Note that $K_{D}^{\prime}$ is finite for $2<D<4$; in particular, $K_{3}^{\prime}=$ $\pi^{2} / 4$. Substituting Eq. (4.3) into Eq. (4.2) and taking the limit $\Lambda_{0} \rightarrow \infty$, we can scale out the $k$-dependence and obtain for $D<3<4$, using the definitions $u_{0}=$ $K_{D} T \Lambda_{0}^{-\epsilon} \rho_{0}^{-2} \Gamma_{0}$ and $k_{c}=\Lambda_{0}\left(u_{0} / u_{*}\right)^{1 / \epsilon}$,

$$
\rho_{0}^{-1}[\Sigma(\mathbf{k})-\Sigma(0)]=B_{D} k_{c}^{2 \epsilon} k^{2-2 \epsilon},
$$

where after an integration by parts the coefficient $B_{D}$ can be cast into the following form,

$$
\begin{gathered}
B_{D}=\frac{3 u_{*}^{2}}{4(D-3)} K_{D}^{\prime} I_{D} \\
I_{D}=\frac{\Omega_{D-1}}{\Omega_{D}} \int_{0}^{\pi} d \vartheta(\sin \vartheta)^{D-2} \int_{0}^{\infty} d x x^{2 D-6} \\
\times\left[-\frac{d}{d x}\left(\frac{1+2 x \cos \vartheta}{1+2 x \cos \vartheta+x^{2}}\right)\right] .
\end{gathered}
$$

In Fig. 3] we show a numerical evaluation of $B_{D}$ for $3<$ $D<4$. With $I_{3}=1$ and $K_{3}^{\prime}=\pi^{2} / 4$ we obtain for $D \rightarrow 3$

$$
B_{D} \sim \frac{b_{3}}{D-3}, b_{3}=\frac{3 \pi^{2} u_{*}^{2}}{16}
$$

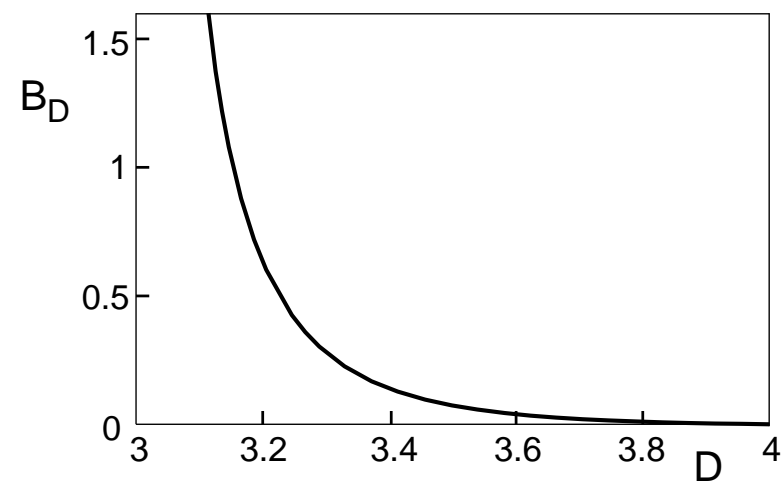

FIG. 3: Graph of the coefficient $B_{D}$ defined in Eqs. (4.6) and (4.7) in dimensions $3<D \leq 4$.

Perturbation theory is valid as long as the correction 4.5 is small compared with $\rho_{0} k^{2}$, i. e. for $k \gtrsim k_{c} B_{D}^{1 / 2 \epsilon}$. Due to the IR divergence of $B_{D}$ for $D \rightarrow 3$, close to three dimensions perturbation theory breaks down even outside the critical regime. In a IR regularized approach with a cutoff $\xi$, the divergence in Eq. (4.5) is replaced by a $\ln (k \xi)$ behavior [5]. Below we shall see that the functional RG approach shows a $\ln \left(k / k_{c}\right)$ behavior, as expected for a theory where the divergence due to density fluctuations is screened within a non-perturbative treatment.

\section{B. Functional RG calculation of the self-energy}

We now derive the behavior of the self-energy microscopically using the functional RG equations for the effective classical field theory.

\section{The $u_{l}^{2}$-truncation for the $\dot{\Gamma}_{l}^{(4)}$ inhomogeneity}

To begin with, we need the RG flow of the fourpoint vertex. In the simplest approximation, we expand $\dot{\Gamma}_{l}^{(4)}\left(\mathbf{q}_{1}^{\prime}, \mathbf{q}_{2}^{\prime} ; \mathbf{q}_{2}, \mathbf{q}_{1}\right)$ in powers of $u_{l}$, which should be accurate as long as the renormalized coupling remains small. To leading order we simply ignore the six-point vertex and replace the four-point vertices on the right-hand side of Eq. (3.15) by their relevant part $u_{l}$. In this approximation,

$$
\begin{array}{r}
\dot{\Gamma}_{l}^{(4)}\left(\mathbf{q}_{1}^{\prime}, \mathbf{q}_{2}^{\prime} ; \mathbf{q}_{2}, \mathbf{q}_{1}\right) \approx-u_{l}^{2}\left[\frac{1}{2} \dot{\chi}_{l}\left(\left|\mathbf{q}_{1}+\mathbf{q}_{2}\right|\right)\right. \\
\left.+\dot{\chi}_{l}\left(\left|\mathbf{q}_{1}-\mathbf{q}_{1}^{\prime}\right|\right)+\dot{\chi}_{l}\left(\left|\mathbf{q}_{1}-\mathbf{q}_{2}^{\prime}\right|\right)\right],
\end{array}
$$

where the generalized susceptibility is given by 


$$
\begin{aligned}
\dot{\chi}_{l}(q) & =2 \int_{\mathbf{q}^{\prime}} \dot{G}\left(\left|\mathbf{q}^{\prime}\right|\right) \tilde{G}_{l}\left(\left|\mathbf{q}^{\prime}+\mathbf{q}\right|\right) \\
& \approx \frac{2}{1+r_{l}}\left\langle\frac{\Theta\left(1<\left|\hat{\mathbf{q}}^{\prime}+\mathbf{q}\right|<e^{l}\right)}{\left|\hat{\mathbf{q}}^{\prime}+\mathbf{q}\right|^{2}+r_{l}}\right\rangle_{\hat{\mathbf{q}}^{\prime}} \\
& =\frac{2}{1+r_{l}} \frac{\Omega_{D-1}}{\Omega_{D}} \int_{0}^{\pi} d \vartheta(\sin \vartheta)^{D-2} \\
& \times \frac{\Theta\left(0<q^{2}+2 q \cos \vartheta<e^{2 l}-1\right)}{1+r_{l}+q^{2}+2 q \cos \vartheta}
\end{aligned}
$$

where in the second and third line we have used the approximations (3.24) and (3.25), taking into account only the relevant and marginal part of the two-point vertex.

\section{Generalized susceptibility}

It is instructive to examine the behavior of the generalized susceptibility $\dot{\chi}_{l}(q)$. For $e^{l}-1 \gg 2$ the asymptotic behavior for small and large $q$ can be easily obtained analytically,

$$
\dot{\chi}_{l}(q) \sim \begin{cases}\frac{1}{\left(1+r_{l}\right)^{2}}+O(q) & \text { for } q \ll 1 \ll e^{l} \\ \frac{2}{\left(1+r_{l}\right) q^{2}} & \text { for } 1 \ll q \ll e^{l} .\end{cases}
$$

The important point is that the leading correction for small $q$ is linear in $q$ : expanding Eq. (4.10) in powers of $q$

$$
\dot{\chi}_{l}(q)=\dot{\chi}_{l}(0)+\dot{\chi}_{l}^{\prime}(0) q+O\left(q^{2}\right)
$$

we find

$$
\begin{gathered}
\dot{\chi}_{l}(0)=\frac{1}{\left(1+r_{l}\right)^{2}}, \\
\dot{\chi}_{l}^{\prime}(0)=-\frac{1}{\left(1+r_{l}\right)^{2}}\left[\frac{4 S_{1}}{\left(1+r_{l}\right)}-S_{0}\right],
\end{gathered}
$$

where

$$
S_{0}=\left\langle\delta\left(\hat{\mathbf{q}}^{\prime} \cdot \hat{\mathbf{q}}\right)\right\rangle_{\hat{\mathbf{q}}^{\prime}}=\frac{\Omega_{D-1}}{\Omega_{D}}
$$

and

$$
S_{1}=\left\langle\Theta\left(\hat{\mathbf{q}}^{\prime} \cdot \hat{\mathbf{q}}\right) \hat{\mathbf{q}}^{\prime} \cdot \hat{\mathbf{q}}\right\rangle_{\hat{\mathbf{q}}^{\prime}}=\frac{S_{0}}{D-1}
$$

In $D=3$ we obtain with $S_{0}=1 / 2$ and $S_{1}=1 / 4$

$$
\dot{\chi}_{l}^{\prime}(0)=-\frac{1}{\left(1+r_{l}\right)^{2}}\left(\frac{1}{1+r_{l}}-\frac{1}{2}\right), \quad D=3 .
$$

For later reference, let us give the exact function $\dot{\chi}_{l}(q)$ in $D=3$, where the angular integration in Eq. (4.10) can easily be performed analytically. The result can be written as

$$
\dot{\chi}_{l}(q)=\frac{\Theta\left(q_{2}-q_{1}\right)}{2\left(1+r_{l}\right) q} \ln \left[\frac{1+r_{l}+q^{2}+2 q q_{2}}{1+r_{l}+q^{2}+2 q q_{1}}\right],
$$

where

$$
\begin{aligned}
& q_{1}=\left\{\begin{array}{ll}
-1 & \text { if } q>2 \\
-q / 2 & \text { if } q<2
\end{array},\right. \\
& q_{2}=\left\{\begin{array}{ll}
1 & \text { if } e^{l}-1>q \\
\frac{e^{2 l}-1}{2 q}-\frac{q}{2} & \text { if } e^{l}-1<q
\end{array} .\right.
\end{aligned}
$$

In particular, for $q \leq \min \left\{2, e^{l}-1\right\}$ we have

$$
\dot{\chi}_{l}(q)=\frac{1}{2\left(1+r_{l}\right) q} \ln \left[\frac{(1+q)^{2}+r_{l}}{1+r_{l}}\right] \text {. }
$$

Thus, for small momenta our approximation (4.9) yields in $D=3$,

$$
\begin{aligned}
& \dot{\Gamma}_{l}^{(4)}\left(\mathbf{q}_{1}^{\prime}, \mathbf{q}_{2}^{\prime} ; \mathbf{q}_{2}, \mathbf{q}_{1}\right) \approx-\frac{5}{2} \frac{u_{l}^{2}}{\left(1+r_{l}\right)^{2}} \\
& \quad+\frac{u_{l}^{2}}{\left(1+r_{l}\right)^{2}}\left(\frac{1}{1+r_{l}}-\frac{1}{2}\right)\left[\frac{1}{2}\left|\mathbf{q}_{1}+\mathbf{q}_{2}\right|\right. \\
& \left.\quad+\left|\mathbf{q}_{1}-\mathbf{q}_{1}^{\prime}\right|+\left|\mathbf{q}_{1}-\mathbf{q}_{2}^{\prime}\right|\right]+O\left(\mathbf{q}_{i}^{2}\right) .
\end{aligned}
$$

The term linear in $q$ generates marginal parameters, as mentioned in SecIIB2 when we iterate the RG. The linear term exists for all $D$, but the corresponding coupling parameters are irrelevant in $D>3$. Because these coupling parameters are not consistently taken into account in the $u^{2}$-truncation given in Eq. (4.9), we cannot expect that this truncation gives numerically accurate results in the critical regime close to three dimensions. On the other hand, in the short-wavelength regime (and also for $D-3 \gg \eta$ ), our $u^{2}$-truncation (4.9) is sufficient. In this section we shall therefore proceed with this approximation, which produces well-defined results even in $D=3$ provided the anomalous exponent $\eta$ is calculated self-consistently by solving an integral equation, see Eq. (4.29) below. In Sec. VI we shall improve on this approximation by explicitly including all marginal coupling parameters in the critical regime in $D=3$.

\section{Explicit expression for the four-point vertex}

With Eq. 4.9 as an approximation for $\dot{\Gamma}_{l}^{(4)}$, we are now in a position to calculate the flow of the four-point vertex. Let us rewrite Eq. (4.9) in the following way,

$$
\dot{\Gamma}_{l}^{(4)}\left(\mathbf{q}_{1}^{\prime}, \mathbf{q}_{2}^{\prime} ; \mathbf{q}_{2}, \mathbf{q}_{1}\right) \approx \frac{-5 u_{l}^{2}}{2\left(1+r_{l}\right)^{2}}+\dot{\Gamma}_{l}^{(4 \mathrm{mi})}\left(\mathbf{q}_{1}^{\prime}, \mathbf{q}_{2}^{\prime} ; \mathbf{q}_{2}, \mathbf{q}_{1}\right)
$$


where

$$
\begin{aligned}
\dot{\Gamma}_{l}^{(4 \mathrm{mi})} & \left(\mathbf{q}_{1}^{\prime}, \mathbf{q}_{2}^{\prime} ; \mathbf{q}_{2}, \mathbf{q}_{1}\right)=-u_{l}^{2}\left[\frac{1}{2} \dot{\chi}_{l}^{(\mathrm{mi})}\left(\left|\mathbf{q}_{1}+\mathbf{q}_{2}\right|\right)\right. \\
& \left.+\dot{\chi}_{l}^{(\mathrm{mi})}\left(\left|\mathbf{q}_{1}-\mathbf{q}_{1}^{\prime}\right|\right)+\dot{\chi}_{l}^{(\mathrm{mi})}\left(\left|\mathbf{q}_{1}-\mathbf{q}_{2}^{\prime}\right|\right)\right], \quad(4
\end{aligned}
$$

with

$$
\dot{\chi}_{l}^{(\mathrm{mi})}(q)=\dot{\chi}_{l}(q)-\dot{\chi}_{l}(0)
$$

describing the momentum-dependent part. The superscript (mi) indicates that these terms are marginal or irrelevant. In this approximation the four-point vertex takes the form

$$
\tilde{\Gamma}_{l}^{(4)}\left(\mathbf{q}_{1}^{\prime}, \mathbf{q}_{2}^{\prime} ; \mathbf{q}_{2}, \mathbf{q}_{1}\right) \approx u_{l}+\tilde{\Gamma}_{l}^{(4 \mathrm{mi})}\left(\mathbf{q}_{1}^{\prime}, \mathbf{q}_{2}^{\prime} ; \mathbf{q}_{2}, \mathbf{q}_{1}\right),
$$

with the irrelevant (and in $D=3$ also marginal) parts given by

$$
\begin{aligned}
\tilde{\Gamma}_{l}^{(4 \mathrm{mi})} & \left(\mathbf{q}_{1}^{\prime}, \mathbf{q}_{2}^{\prime} ; \mathbf{q}_{2}, \mathbf{q}_{1}\right)=\int_{0}^{l} d l^{\prime} e^{\epsilon\left(l-l^{\prime}\right)-2 \int_{l^{\prime}}^{l} d \tau \eta_{\tau}} \dot{\Gamma}_{l^{\prime}}^{(4 \mathrm{mi})}\left(e^{-\left(l-l^{\prime}\right)} \mathbf{q}_{1}^{\prime}, e^{-\left(l-l^{\prime}\right)} \mathbf{q}_{2}^{\prime} ; e^{-\left(l-l^{\prime}\right)} \mathbf{q}_{2}, e^{-\left(l-l^{\prime}\right)} \mathbf{q}_{1}\right) \\
= & -\int_{0}^{l} d l^{\prime} e^{\epsilon\left(l-l^{\prime}\right)-2 \int_{l^{\prime}}^{l} d \tau \eta_{\tau}} u_{l^{\prime}}^{2}\left[\frac{1}{2} \dot{\chi}_{l^{\prime}}^{(\mathrm{mi})}\left(e^{-\left(l-l^{\prime}\right)}\left|\mathbf{q}_{1}+\mathbf{q}_{2}\right|\right)+\dot{\chi}_{l^{\prime}}^{(\mathrm{mi})}\left(e^{-\left(l-l^{\prime}\right)}\left|\mathbf{q}_{1}-\mathbf{q}_{1}^{\prime}\right|\right)+\dot{\chi}_{l^{\prime}}^{(\mathrm{mi})}\left(e^{-\left(l-l^{\prime}\right)}\left|\mathbf{q}_{1}-\mathbf{q}_{2}^{\prime}\right|\right)\right] \\
= & -\int_{0}^{l} d t e^{\epsilon t-2 \int_{l-t}^{l} d \tau \eta_{\tau}} u_{l-t}^{2}\left[\frac{1}{2} \dot{\chi}_{l-t}^{(\mathrm{mi})}\left(e^{-t}\left|\mathbf{q}_{1}+\mathbf{q}_{2}\right|\right)+\dot{\chi}_{l-t}^{(\mathrm{mi})}\left(e^{-t}\left|\mathbf{q}_{1}-\mathbf{q}_{1}^{\prime}\right|\right)+\dot{\chi}_{l-t}^{(\mathrm{mi})}\left(e^{-t}\left|\mathbf{q}_{1}-\mathbf{q}_{2}^{\prime}\right|\right)\right]
\end{aligned}
$$

\section{Anomalous dimension}

To obtain the anomalous dimension $\eta_{l}=$ $\partial \dot{\Gamma}_{l}^{(2)}(\mathbf{q}) /\left.\partial q^{2}\right|_{q^{2}=0}$ we calculate $\dot{\Gamma}_{l}^{(2)}(\mathbf{q})$ via Eq. (3.12). With the approximation Eqs. 4.26 4.27) for the four-point vertex we find

$$
\begin{aligned}
& \dot{\Gamma}_{l}^{(2)}(\mathbf{q})=\frac{1}{R_{l}(1)}\left\langle\tilde{\Gamma}_{l}^{(4)}\left(\mathbf{q}, \hat{\mathbf{q}}^{\prime} ; \hat{\mathbf{q}}^{\prime}, \mathbf{q}\right)\right\rangle_{\hat{\mathbf{q}}^{\prime}} \\
& \approx \frac{u_{l}}{1+r_{l}}-\frac{3}{2\left(1+r_{l}\right)} \int_{0}^{l} d t e^{\epsilon t-2 \int_{l-t}^{l} d \tau \eta_{\tau}} u_{l-t}^{2} \\
& \times\left\langle\dot{\chi}_{l-t}^{(\mathrm{mi})}\left(e^{-t}\left(\left|\hat{\mathbf{q}}^{\prime}+\mathbf{q}\right|\right)\right\rangle_{\hat{\mathbf{q}}^{\prime}} .\right.
\end{aligned}
$$

From Eq. (3.21) we then obtain an integral equation for the flowing anomalous dimension

$$
\eta_{l}=\int_{0}^{l} d t K(l, t) u_{l-t}^{2} e^{-2 \int_{l-t}^{l} d \tau \eta_{\tau}},
$$

with

$$
\begin{aligned}
& K(l, t)=-\left.\frac{3 e^{\epsilon t}}{2\left(1+r_{l}\right)} \frac{\partial}{\partial q^{2}}\left\langle\dot{\chi}_{l-t}\left(e^{-t}\left(\left|\hat{\mathbf{q}}^{\prime}+\mathbf{q}\right|\right)\right)\right\rangle_{\hat{\mathbf{q}}^{\prime}}\right|_{q=0} \\
&=-\frac{3}{4 D\left(1+r_{l}\right)}\left[(D-1) e^{-(D-3) t} \dot{\chi}_{l-t}^{\prime}\left(e^{-t}\right)\right. \\
&\left.\quad+e^{-(D-2) t} \dot{\chi}_{l-t}^{\prime \prime}\left(e^{-t}\right)\right],
\end{aligned}
$$

where $\dot{\chi}_{l}^{\prime}(x)=d \dot{\chi}_{l}(x) / d x$ and $\dot{\chi}_{l}^{\prime \prime}(x)=d^{2} \dot{\chi}_{l}(x) / d x^{2}$. Note that on the right-hand side we have replaced $\dot{\chi}_{l-t}^{(\mathrm{mi})}(q) \rightarrow \dot{\chi}_{l-t}(q)$, because the constant part $\dot{\chi}_{l-t}(0)$ does not contribute to the derivative. The integral equation (4.29) together with the one-loop flow equations for the relevant coupling parameters,

$$
\partial_{l} r_{l}=\left(2-\eta_{l}\right) r_{l}+\frac{u_{l}}{1+r_{l}},
$$

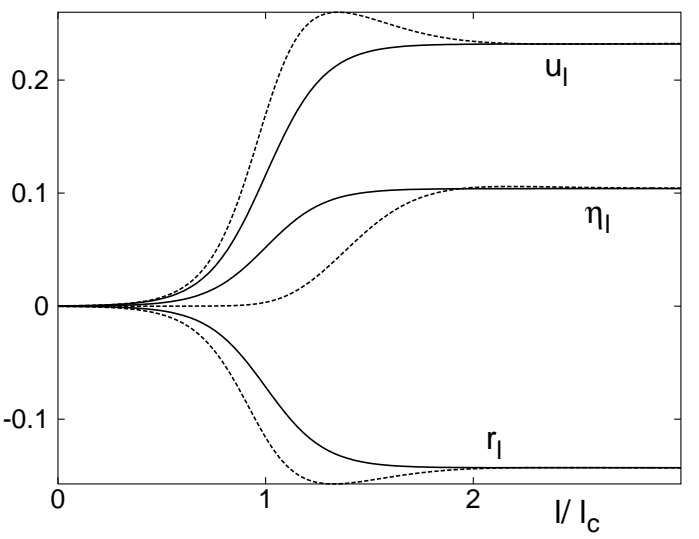

FIG. 4: Self-consistent RG flows of $u_{l}, r_{l}$ and $\eta_{l}$ at the critical point in $D=3$ (dashed lines), obtained numerically from the coupled intego-differential Eqs. 4.29 4.314.32). For comparison, the Fermi-function approximation Eq. 4.33) for the flows is also shown (full lines), with fixed point values matching the numerical solution.

$$
\partial_{l} u_{l}=\left(\epsilon-2 \eta_{l}\right) u_{l}-\frac{5}{2} \frac{u_{l}^{2}}{\left(1+r_{l}\right)^{2}},
$$

form a system of three coupled integro-differential equations for the three unknown functions $r_{l}, u_{l}$, and $\eta_{l}$. A numerical solution of these equations for $D=3$ is shown in Fig. 4

To leading order in $\epsilon$ (i. e. close to four dimensions) Eqs. (4.294.314.32) decouple and one recovers the fixed point values $u_{*}=2 \epsilon / 5, r_{*}=-\epsilon / 5$ from Eq. (3.37). To this order it is consistent to ignore $\eta_{l}$ and $r_{l}$ on 
the right-hand side of Eq. (4.32), so that it reduces to Eq. (3.29), with the Fermi function solution for $u_{l}$ given in Eq. (3.31). From the numerical solution of Eqs. 4.29 4.32) for $D=3$ we find that even if $r_{l}$ and $\eta_{l}$ are taken into account, to a good approximation the qualitative behavior of $u_{l}$ is still well described by a Fermi function if we adjust the fixed point value $u_{*}$ to the one obtained from the numerical solution. Furthermore, Fig. 4 shows that also $\eta_{l}$ and $r_{l}$ roughly follow the same functional form and we therefore approximate

$$
\frac{\eta_{l}}{\eta} \approx \frac{r_{l}}{r_{*}} \approx \frac{u_{l}}{u_{*}} \approx \frac{1}{e^{\epsilon\left(l_{c}-l\right)}+1},
$$

with the fixed point values taken from the numerical solution and where $\eta=\lim _{l \rightarrow \infty} \eta_{l}$ is the anomalous dimension at the fixed point. Although the functional form (4.33) for $\eta_{l}$ is qualitatively incorrect for $l \ll l_{c}$ (where $\eta_{l} \propto u_{l}^{2}$, whereas Eq. (4.33) predicts $\eta_{l} \propto u_{l}$ ) we may use Eq. (4.33) as a zeroth approximation on the right-hand side of the integral equation (4.29). The point is that for $l \ll l_{c}$ the right-hand side of Eq. (4.29) is not sensitive to the precise value of $\eta_{l}$ in this regime, and the first iteration of the integral equation yields an accurate result even for small $l$. Let us now calculate the fixed point values. We obtain from Eq. (4.29)

$$
\eta=u_{*}^{2} \int_{0}^{\infty} d t K(\infty, t) e^{-2 \eta t}
$$

where we use the notation $\eta=\eta_{*}$. The function $K(\infty, t)$ is obtained from $K(l, t)$ defined in Eq. (4.30) by replacing the functions $\dot{\chi}_{l-t}(q)$ on the right-hand side by

$$
\begin{aligned}
\dot{\chi}_{*}(q) & \equiv \lim _{l \rightarrow \infty} \dot{\chi}_{l}(q) \\
& \approx \frac{2}{1+r_{*}}\left\langle\frac{\Theta\left(\left|\hat{\mathbf{q}}^{\prime}+\mathbf{q}\right|-1\right)}{\left|\hat{\mathbf{q}}^{\prime}+\mathbf{q}\right|^{2}+r_{*}}\right\rangle_{\hat{\mathbf{q}}^{\prime}} .
\end{aligned}
$$

In particular, in $D=3$ we have

$$
\begin{array}{r}
\dot{\chi}_{*}(q)=\frac{1}{2\left(1+r_{*}\right) q}\left[\Theta(2-q) \ln \left(\frac{(1+q)^{2}+r_{*}}{1+r_{*}}\right)\right. \\
\left.+\Theta(q-2) \ln \left(\frac{(1+q)^{2}+r_{*}}{(1-q)^{2}+r_{*}}\right)\right] .
\end{array}
$$

Note that for large $t$ the kernel $K(\infty, t)$ vanishes as $e^{-(D-3) t}$, so that in $D=3$ it is crucial to retain the $\eta$-dependence on the right-hand side of Eq. (4.34). This is closely related to the appearance of marginal terms of the four-point vertex in $D=3$. The self-consistent fixed point values can be obtained by using the fact that, at the fixed point, we can express $u_{*}$ and $r_{*}$ as a function of $\eta$,

$$
\begin{aligned}
& r_{*}=-\frac{2}{3} \frac{1-2 \eta}{4-3 \eta} \\
& u_{*}=\frac{10}{9} \frac{(1-2 \eta)(2-\eta)^{2}}{(4-3 \eta)^{2}} .
\end{aligned}
$$

Inserting these expressions for $u_{*}$ and $r_{*}$ into Eq. (4.34), we obtain a self-consistent equation for $\eta$ which can be solved numerically. The numerical solution gives

$$
\begin{aligned}
r_{*} & \approx-0.143 \\
u_{*} & \approx 0.232, \\
\eta & \approx 0.104
\end{aligned}
$$

The above value for $\eta$ is approximately three times as large as the generally accepted value $\eta \approx 0.038$ obtained by several different techniques [22, 23]. However, given the simplicity of our truncation, it is quite satisfactory that our estimate (4.38c) for $\eta$ has the correct order of magnitude. In any case, our simple $u^{2}$-truncation certainly gives a much better estimate for $\eta$ than the selfconsistent two-loop calculation employed by Baym et al. [5], which predicts a value of 0.5 for $\eta$. In Sec VI we shall further improve on the $u^{2}$-truncation employed here by explicitly taking the RG flow of marginal coupling parameters into account.

For completeness, we show how to obtain the standard result for $\eta$ to second order in an $\epsilon$-expansion. We can rewrite Eq. (4.34) as

$$
\begin{aligned}
\eta & =-\frac{3 u_{*}^{2}}{4 D\left(1+r_{*}\right)} \int_{0}^{1} d \lambda \lambda^{-\epsilon+2 \eta}\left[(D-1) \dot{\chi}_{*}^{\prime}(\lambda)+\lambda \dot{\chi}_{*}^{\prime \prime}(\lambda)\right] \\
& =-\frac{3 u_{*}^{2}}{4 D\left(1+r_{*}\right)}\left[2(1-\eta) \int_{0}^{1} d \lambda \lambda^{-\epsilon+2 \eta} \dot{\chi}_{*}^{\prime}(\lambda)+\dot{\chi}_{*}^{\prime}(1)\right] .
\end{aligned}
$$

For small $\epsilon=4-D$ we obtain to leading order

$$
\eta=-\frac{3 u_{*}^{2}}{16}\left[2(\dot{\chi}(1)-\dot{\chi}(0))+\dot{\chi}^{\prime}(1)\right],
$$

where the function $\dot{\chi}(q)$ is obtained from $\dot{\chi}_{*}(q)$ by simply setting $r_{*} \rightarrow 0$. Evaluating the integrals in $D=4$ (see also Ref. [12]),

$$
\begin{aligned}
\dot{\chi}(0) & =1, \\
\dot{\chi}(1) & =\frac{4}{3}-\frac{\sqrt{3}}{\pi}, \\
\dot{\chi}^{\prime}(1) & =-\frac{4}{3}+\frac{2 \sqrt{3}}{\pi},
\end{aligned}
$$

and using $u_{*}=2 \epsilon / 5$, we finally obtain $\eta=\epsilon^{2} / 50+O\left(\epsilon^{3}\right)$, in agreement with the field theoretical result [21].

\section{The scaling function}

Let us now calculate the dimensionless scaling function $\sigma_{l}(x)$ which we define by

$$
\sigma_{l}(x)=e^{-2\left(l-l_{c}\right)} Z_{l}^{-1} \tilde{\Gamma}_{l}^{(2)}\left(e^{\left(l-l_{c}\right)} x\right) .
$$

Since the two-point vertex $\tilde{\Gamma}_{l}^{(2)}(\mathbf{q})$ and the inhomogeneity $\dot{\Gamma}_{l}^{(2)}(\mathbf{q})$ depend only on $q=|\mathbf{q}|$, we shall in this subsection 
use scalar arguments for these functions to simplify the notation. For $l \rightarrow \infty$ the definition (4.42) for $\sigma_{l}(x)$ yields the universal scaling function defined in Eq. (1.2),

$$
\lim _{l \rightarrow \infty} \sigma_{l}(x) \equiv \sigma(x)=\left(\rho_{0} k_{c}^{2}\right)^{-1}\left[\Sigma\left(k_{c} x\right)-\Sigma(0)\right] .
$$

We first perform some exact manipulations. The functional flow equation for $\tilde{\Gamma}_{l}^{(2)}(q)$ given by Eq. 3.11) can be transformed into an integral equation

$$
\begin{aligned}
\tilde{\Gamma}_{l}^{(2)}(q)= & e^{2 l-\int_{0}^{l} d \tau \eta_{\tau}} \tilde{\Gamma}_{l=0}^{(2)}\left(e^{-l} q\right) \\
& +\int_{0}^{l} d t e^{2 t-\int_{l-t}^{l} d \tau \eta_{\tau}} \dot{\Gamma}_{l-t}^{(2)}\left(e^{-t} q\right) \\
= & e^{2 l-\int_{0}^{l} d \tau \eta_{\tau}}\left[\tilde{\Gamma}_{l=0}^{(2)}\left(e^{-l} q\right)\right. \\
+ & \left.\int_{0}^{l} d l^{\prime} e^{-2 l^{\prime}+\int_{0}^{l^{\prime}} d \tau \eta_{\tau}} \dot{\Gamma}_{l^{\prime}}^{(2)}\left(e^{-\left(l-l^{\prime}\right)} q\right)\right]
\end{aligned}
$$

To describe a critical system, we choose the initial value $\tilde{\Gamma}_{l=0}^{(2)}(q)=r_{0}$ to be momentum independent such that for $l \rightarrow \infty$ the relevant coupling

$$
r_{l}=e^{2 l-\int_{0}^{l} d \tau \eta_{\tau}}\left[r_{0}+\int_{0}^{l} d l^{\prime} e^{-2 l^{\prime}+\int_{0}^{l^{\prime}} d \tau \eta_{\tau}} \dot{\Gamma}_{l^{\prime}}^{(2)}(0)\right]
$$

has a finite limit $r_{*}=\lim _{l \rightarrow \infty} r_{l}$, just has we have done in Eq. (3.34). This is guaranteed if the initial value $r_{0}$ is chosen such that 24]

$$
r_{0}=-\int_{0}^{\infty} d l^{\prime} e^{-2 l^{\prime}+\int_{0}^{l^{\prime}} d \tau \eta_{\tau}} \dot{\Gamma}_{l^{\prime}}^{(2)}(0)
$$

Defining

$$
\dot{\Gamma}_{l}^{(2 \mathrm{mi})}(q)=\dot{\Gamma}_{l}^{(2)}(q)-\dot{\Gamma}_{l}^{(2)}(0),
$$

where the superscript $m i$ indicates that these function contain only marginal and irrelevant parameters, we obtain

$$
\begin{aligned}
& \sigma_{l}(x)=e^{-2\left(l-l_{c}\right)} Z_{l}^{-1} r_{l} \\
& \quad+\int_{0}^{l} d l^{\prime} e^{-2\left(l^{\prime}-l_{c}\right)+\int_{0}^{l^{\prime}} d \tau \eta_{\tau}} \dot{\Gamma}_{l^{\prime}}^{(2 \mathrm{mi})}\left(e^{l^{\prime}-l_{c}} x\right) .
\end{aligned}
$$

The first term on the right hand side vanishes for $l \rightarrow \infty$ because by construction $\lim _{l \rightarrow \infty} r_{l}=r_{*}$ is finite on the critical surface. Taking the limit $l \rightarrow \infty$ we thus obtain (after renaming $l^{\prime} \rightarrow l$ )

$$
\sigma(x)=\int_{0}^{\infty} d l e^{-2\left(l-l_{c}\right)+\int_{0}^{l} d \tau \eta_{\tau}} \dot{\Gamma}_{l}^{(2 \mathrm{mi})}\left(e^{l-l_{c}} x\right) .
$$

\section{Truncation of the flow equations}

So far, no approximation has been made. We now approximate the function $\dot{\Gamma}_{l}^{(2 \mathrm{mi})}(q)$ on the right-hand side of Eq. (4.49) by the leading term in the expansion in powers of $u_{l}$, see Eq. (4.28,

$$
\begin{aligned}
\dot{\Gamma}_{l}^{(2 \mathrm{mi})}(q) \approx & -\frac{3}{2\left(1+r_{l}\right)} \int_{0}^{l} d t e^{\epsilon t-2 \int_{l-t}^{l} d \tau \eta_{\tau}} u_{l-t}^{2} \\
& \times\left\langle\dot{\chi}_{l-t}\left(e^{-t}\left|\hat{\mathbf{q}}^{\prime}+\mathbf{q}\right|\right)-\dot{\chi}_{l-t}\left(e^{-t}\right)\right\rangle_{\hat{\mathbf{q}}^{\prime}} \\
= & -\frac{3}{2\left(1+r_{l}\right)} \int_{0}^{l} d l^{\prime} e^{\epsilon\left(l-l^{\prime}\right)-2 \int_{l^{\prime}}^{l} d \tau \eta_{\tau}} u_{l^{\prime}}^{2} \\
& \times\left\langle\dot{\chi}_{l^{\prime}}\left(e^{-\left(l-l^{\prime}\right)}\left|\hat{\mathbf{q}}^{\prime}+\mathbf{q}\right|\right)-\dot{\chi}_{l^{\prime}}\left(e^{-\left(l-l^{\prime}\right)}\right)\right\rangle_{\hat{\mathbf{q}}^{\prime}} .
\end{aligned}
$$

All quantities on the right-hand side of Eq. (4.49) are now known, so that we may calculate the crossover function by performing the four-dimensional integration (two angular integrations and two integrations over the scale parameters $l$ and $l^{\prime}$ ) numerically. There are no divergences even in $D=3$ provided the integral equation (4.29) for $\eta_{l}$ is solved self-consistently.

\section{Results for the scaling function}

To obtain an analytic approximation for the scaling function $\sigma(x)$, we adopt again the successful strategy used in the solution of the integral equation (4.29) for $\eta$ : we substitute the Fermi function ansatz (4.33) for the flowing anomalous dimension $\eta_{\tau}$ on the right-hand side of Eqs. (4.49) and (4.50). After some transformations of the integration variables we then obtain

$$
\sigma(x)=\frac{3 u_{*}^{2}}{2} x^{2-\eta} \int_{x e^{-l_{c}}}^{\infty} d y \frac{y^{-3+2 \epsilon}}{\left[x^{\epsilon}+y^{\epsilon}\right]^{2-2 \eta / \epsilon}} F\left(x, y ; \eta, l_{c}\right)
$$

with

$$
\begin{aligned}
& F\left(x, y ; \eta, l_{c}\right)=\int_{0}^{1} d z \frac{z^{1-\epsilon}}{\left[x^{\epsilon}+(y / z)^{\epsilon}\right]^{\eta / \epsilon}\left[1+r_{l_{c}+\ln \frac{y}{z x}}\right]} \\
& \quad \times\left\langle\dot{\chi}_{l_{c}+\ln \frac{y}{x}}(z)-\dot{\chi}_{l_{c}+\ln \frac{y}{x}}\left(\left|z \hat{\mathbf{q}}^{\prime}+y \hat{\mathbf{q}}^{\prime \prime}\right|\right)\right\rangle_{\hat{\mathbf{q}}^{\prime}},
\end{aligned}
$$

where $\hat{\mathbf{q}}^{\prime \prime}$ is an arbitrary unit vector. Note that by assumption $x \ll e^{l_{c}}$ so that we may replace $x e^{-l_{c}} \rightarrow 0$ in the lower limit of the $y$-integral in Eq. 4.51). Consider first the regime $x \ll 1$. Using the fact that for $l \gg l_{c}$ we may approximate $r_{l} \rightarrow r_{*}$ and $\dot{\chi}_{l}(q) \rightarrow \lim _{l \rightarrow \infty} \dot{\chi}_{l}(q) \equiv$ $\dot{\chi}_{*}(q)$ (see Eq. (4.35), we may replace in this regime $\dot{\chi}_{l_{c}+\ln \frac{y}{x}}(q) \rightarrow \dot{\chi}_{*}(q)$ and $r_{l_{c}+\ln \frac{y}{z x}} \rightarrow r_{*}$ in Eq. (4.52). Then we obtain

$$
\sigma(x) \approx \frac{3 u_{*}^{2}}{2} x^{2-\eta} \int_{0}^{\infty} d y \frac{y^{-3+2 \epsilon}}{\left[x^{\epsilon}+y^{\epsilon}\right]^{2-2 \eta / \epsilon}} F_{*}(x, y ; \eta) .
$$

Using $D$-dimensional spherical coordinates, the function $F_{*}(x, y ; \eta) \equiv \lim _{l_{c} \rightarrow \infty} F\left(x, y ; \eta, l_{c}\right)$ can be written as

$$
\begin{aligned}
& F_{*}(x, y ;\eta)=\frac{\Omega_{D-1}}{\left(1+r_{*}\right) \Omega_{D}} \int_{0}^{1} d z \int_{0}^{\pi} d \vartheta \frac{z^{1-\epsilon}(\sin \vartheta)^{D-2}}{\left[x^{\epsilon}+(y / z)^{\epsilon}\right]^{\eta / \epsilon}} \\
& \times\left[\dot{\chi}_{*}(z)-\dot{\chi}_{*}\left(\sqrt{z^{2}+2 z y \cos \vartheta+y^{2}}\right)\right] \cdot
\end{aligned}
$$


From Eqs. (4.53) and (4.54) it is now straightforward to obtain the asymptotic behavior of $\sigma(x)$ for small $x$,

$$
\begin{gathered}
\sigma(x) \sim A_{D} x^{2-\eta} \\
A_{D}=\frac{3 u_{*}^{2}}{2} \int_{0}^{\infty} d y y^{-3+2 \eta} F_{*}(0, y ; \eta) \\
=\frac{3 u_{*}^{2}}{2\left(1+r_{*}\right)} \frac{\Omega_{D-1}}{\Omega_{D}} \int_{0}^{\infty} d y y^{-3+\eta} \int_{0}^{1} d z z^{1-\epsilon+\eta} \int_{0}^{\pi} d \vartheta \\
\times(\sin \vartheta)^{D-2}\left[\dot{\chi}_{*}(z)-\dot{\chi}_{*}\left(\sqrt{z^{2}+2 z y \cos \vartheta+y^{2}}\right)\right] .
\end{gathered}
$$

In $D=3$ we find numerically

$$
A_{3} \approx 1.17 \text {. }
$$

On the other hand, for large $x$ the flow parameter in $\dot{\chi}_{l_{c}+\ln \frac{y}{x}}(q)$ and $r_{l_{c}+\ln \frac{y}{z x}}$ is typically small compared with $l_{c}$. To take the effect of the flowing $r_{l}$ approximately into account, we replace in this regime $r_{l_{c}+\ln \frac{y}{z x}} \rightarrow r_{0}$ and $\dot{\chi}_{l_{c}+\ln \frac{y}{x}}(q) \rightarrow \dot{\chi}_{0}(q)$, where $\dot{\chi}_{0}(q)$ is obtained from $\dot{\chi}_{*}(q)$ by replacing $r_{*} \rightarrow r_{0}$. Using Eq. (3.35) one can show that on the critical surface and for small $u_{0}$, the initial value of $r_{0}$ is approximately given by $r_{0} \approx-u_{0} /(2-\epsilon)$. With this approximation we obtain in the regime $[2(D-3)]^{-1} \ll$ $\ln x$,

$$
\sigma(x) \sim B_{D} x^{2(D-3)},
$$

$B_{D}=\frac{3 u_{*}^{2}}{2\left(1+r_{0}\right)} \frac{\Omega_{D-1}}{\Omega_{D}} \int_{0}^{\infty} d y y^{-3+2 \epsilon} \int_{0}^{1} d z z^{1-\epsilon} \int_{0}^{\pi} d \vartheta$

$\times(\sin \vartheta)^{D-2}\left[\dot{\chi}_{0}(z)-\dot{\chi}_{0}\left(\sqrt{z^{2}+2 z y \cos \vartheta+y^{2}}\right)\right]$,

and for $1 \ll \ln x \ll[2(D-3)]^{-1}$ (which includes the limit $D \rightarrow 3)$,

$$
\sigma(x) \sim B_{3}^{\prime} \ln x+B_{3}^{\prime \prime},
$$

where

$$
B_{3}^{\prime}=\frac{3 u_{*}^{2}}{2\left(1+r_{0}\right)} \int_{0}^{1} d z \dot{\chi}_{0}(z) \approx \frac{3 \pi^{2}}{24} u_{*}^{2},
$$

and on the right-hand side we have set $r_{0} \rightarrow 0$. Numerically we find $B_{3}^{\prime \prime} \approx 0.0319$. Note that the physical self-energy

$$
\Sigma(k)-\Sigma(0)=\rho_{0} k_{c}^{2} \sigma\left(k / k_{c}\right)
$$

is independent of $u_{*}^{2}$ in the regime $k \gg k_{c}$ : keeping in mind that $k_{c}=\Lambda_{0}\left(u_{0} / u_{*}\right)^{1 / \epsilon}$, it is obvious that the factor of $u_{*}^{2}$ in Eqs. (4.59) and (4.61) combines with a suitable power of $k_{c}$ so that the self-energy is proportional to $u_{0}^{2}$.

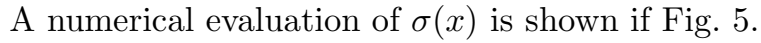

To summarize, using the functional RG, we have derived in this section the momentum-dependent selfenergy of weakly interacting bosons, covering the entire range from the critical regime up to momenta of

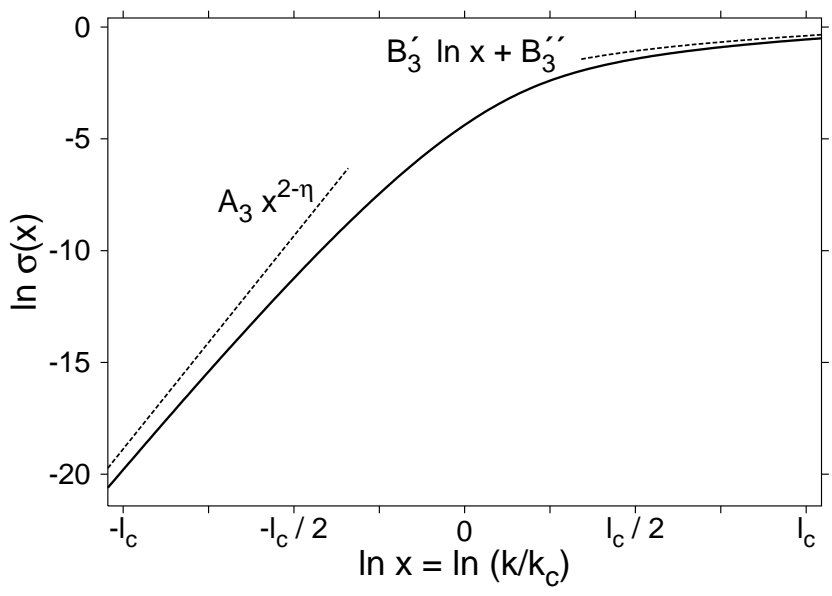

FIG. 5: Numerical evaluation of the scaling function $\sigma(x)$ of the self-energy in the limit $a \rightarrow 0$ and $D=3$. Dashed lines are the asymptotic forms predicted by Eqs. 4.554.60).

the order of the inverse thermal de Broglie wavelength. While in the critical regime $k \ll k_{c}$ it is simpler to obtain the asymptotic long-wavelength behavior of the selfenergy within the field theoretical RG, the functional RG approach adopted in this work can describe the entire crossover from the critical regime $k \ll k_{c}$ to the shortwavelength regime $k_{c} \ll k \lesssim 2 \pi / \lambda_{\text {th }}$. Note that the behavior of the self-energy in the short-wavelength regime is determined by irrelevant terms which are simply discarded in the field theoretical RG.

\section{INTERACTION INDUCED $T_{c}$-SHIFT}

Baym et al. [5] have shown that to lowest order in the scattering length $a$, the interaction induced shift for the critical temperature $T_{c}$ in $D=3$ can be calculated from the k-dependence of the zero frequency self-energy $\Sigma(\mathbf{k}, 0)$. In a straightforward generalization of their result to arbitrary dimensions, we can write the contribution from classical modes to the shift in $T_{c}$ as

$$
\begin{gathered}
\frac{\Delta T_{c}}{T_{c}}=\frac{2 \Omega_{D}}{\pi D \zeta(D / 2)}\left(\frac{k_{c}}{\Lambda_{0}}\right)^{D-2} \\
\times \int_{0}^{\Lambda_{0} / k_{c}} d x x^{D-3} \frac{\left(\rho_{0} k_{c}^{2}\right)^{-1}\left[\Sigma\left(k_{c} x\right)-\Sigma(0)\right]}{x^{2}+\left(\rho_{0} k_{c}^{2}\right)^{-1}\left[\Sigma\left(k_{c} x\right)-\Sigma(0)\right]}
\end{gathered}
$$

Using $k_{c} / \Lambda_{0}=e^{-l_{c}}=\left(u_{0} / u_{*}\right)^{1 / \epsilon}$, and substituting our scaling function $\sigma(x)$ as defined in Eq. (1.2), this can also be written as

$$
\begin{aligned}
\frac{\Delta T_{c}}{T_{c}}= & \frac{2 \Omega_{D}}{\pi D \zeta(D / 2)}\left(\frac{u_{0}}{u_{*}}\right)^{\frac{D-2}{4-D}} \\
& \times \int_{0}^{\Lambda_{0} / k_{c}} d x x^{D-3} \frac{\sigma(x)}{x^{2}+\sigma(x)} .
\end{aligned}
$$


The shift in $T_{c}$ is dominated by classical fluctuations as long as the UV cutoff $\Lambda_{0} / k_{c}$ can be removed in Eq. (5.2). Keeping in mind that according to Eq. (4.58) $\sigma(x) \sim B_{D} x^{2(D-3)}$ for large $x$, we see that the integral (5.1) is UV convergent as long as $\frac{D-2}{4-D}<2$, i. e. $D<10 / 3$. For $D \geq 10 / 3$ the value of the integral in Eq. (5.1) depends on the UV cutoff, such that the lowest order contribution to the shift in $T_{c}$ is proportional to $u_{0}^{2}$, with logarithmic corrections $\left(\propto u_{0}^{2} \ln u_{0}\right)$ in $D=10 / 3$. Hence for $D \geq 10 / 3$ the shift in the critical temperature cannot be obtained from an effective classical field theory, while for $D<10 / 3$, the cutoff only leads to higher order corrections (in $D=3$ one finds $u_{0}^{2}$ and $u_{0}^{2} \ln \left(u_{0}\right)$ corrections 5]). Writing

$$
\frac{\Delta T_{c}}{T_{c}}=J_{D} u_{0}^{\frac{D-2}{4-D}},
$$

we obtain for $D<10 / 3$,

$$
J_{D}=\frac{2 \Omega_{D}}{\pi D \zeta(D / 2)}\left(\frac{1}{u_{*}}\right)^{\frac{D-2}{4-D}} \int_{0}^{\infty} d x x^{D-3} \frac{\sigma(x)}{x^{2}+\sigma(x)} .
$$

Note that $J_{D}$ implicitly depends on $u_{0}$ via $\sigma(x)$. However, for $u_{0} \rightarrow 0$ the coefficient $J_{D}$ approaches a finite limit independent of $u_{0}$ as long as $D<10 / 3$.

In $D=3$ one usually writes

$$
\frac{\Delta T_{c}}{T_{c}}=c_{1} a n^{1 / 3} .
$$

Keeping in mind that

$$
u_{0}=16 \pi^{-1}[\zeta(3 / 2)]^{-1 / 3} a n^{1 / 3} \text { for } D=3 \text {, }
$$

we have in this case $c_{1}=16 \pi^{-1}[\zeta(3 / 2)]^{-1 / 3} J_{3}$. Within our approximation we find $c_{1}=1.23$, in rather good agreement with the most accurate numerical investigations which give $c_{1}=1.30 \pm 0.02[14]$ and $c_{1}=1.29 \pm 0.05$ [15]. Variational perturbation techniques give similar results, $c_{1}=1.23 \pm 0.12[16]$, and $c_{1}=1.27 \pm 0.11$ [17]. More results from other approaches are summarized in a recent review [2]. There is also experimental data from a ${ }^{4} \mathrm{He}-\mathrm{V} y$ cor system confirming the linear scaling of $T_{c}$ with $a n^{1 / 3}[25]$. The reported value $c_{1} \approx 4.66$ seems however rather large which may be attributed to uncertainties regarding the precise value of $a$ in the experiment, see the discussion in Ref. [15].

\section{IMPROVED DESCRIPTION OF THE CRITICAL REGIME IN $D=3$ : INCLUDING MARGINAL TERMS}

Within the $u^{2}$-truncation employed so far, we have neglected the six-point vertex and approximated the inhomogeneity in the flow equation of the four-point vertex by

$$
\begin{array}{r}
\dot{\Gamma}_{l}^{(4)}\left(\mathbf{q}_{1}^{\prime}, \mathbf{q}_{2}^{\prime} ; \mathbf{q}_{2}, \mathbf{q}_{1}\right) \approx-u_{l}^{2}\left[\frac{1}{2} \dot{\chi}_{l}\left(\left|\mathbf{q}_{1}+\mathbf{q}_{2}\right|\right)\right. \\
\left.+\dot{\chi}_{l}\left(\left|\mathbf{q}_{1}-\mathbf{q}_{1}^{\prime}\right|\right)+\dot{\chi}_{l}\left(\left|\mathbf{q}_{1}-\mathbf{q}_{2}^{\prime}\right|\right)\right],
\end{array}
$$

see Eq. (4.9). This approximation amounts to a truncation of the exact hierarchy of flow equations where on the right-hand side of Eq. (3.15) we simply replace

$$
\tilde{\Gamma}_{l}^{(4)}\left(\mathbf{q}_{1}^{\prime}, \mathbf{q}_{2}^{\prime} ; \mathbf{q}_{2}, \mathbf{q}_{1}\right) \rightarrow u_{l}
$$

A formal justification for this procedure can only be given for $D>3$ : for weak interactions, irrelevant coupling parameters can be expanded in powers of relevant and marginal ones [12, 20]. For $3<D<4$ the only part of the four-point vertex which is not irrelevant is its constant part $u_{l}$. However, in $D=3$ there are two additional marginal parameters related to the momentum dependence of the four-point vertex. Hence, in this case Eq. 6.1) is not consistent. For small momenta the flowing four-point vertex has the form

$$
\begin{aligned}
\tilde{\Gamma}_{l}^{(4)}\left(\mathbf{q}_{1}^{\prime}, \mathbf{q}_{2}^{\prime} ; \mathbf{q}_{2}, \mathbf{q}_{1}\right)=u_{l} \\
+a_{l}\left(\left|\mathbf{q}_{1}-\mathbf{q}_{1}^{\prime}\right|+\left|\mathbf{q}_{1}-\mathbf{q}_{2}^{\prime}\right|\right)+b_{l}\left|\mathbf{q}_{1}+\mathbf{q}_{2}\right| \\
+\tilde{\Gamma}_{l}^{(4 \mathrm{i})}\left(\mathbf{q}_{1}^{\prime}, \mathbf{q}_{2}^{\prime} ; \mathbf{q}_{2}, \mathbf{q}_{1}\right),
\end{aligned}
$$

where the irrelevant part $\tilde{\Gamma}_{l}^{(4 \mathrm{i})}\left(\mathbf{q}_{1}^{\prime}, \mathbf{q}_{2}^{\prime} ; \mathbf{q}_{2}, \mathbf{q}_{1}\right)$ vanishes at least quadratically if all momenta become small. Below, we will denote the diagrams entering the renormalization of the four-point vertex using the usual fermionic language (i. e. BCS, ZS and ZS', see Fig. 1). We emphasize that this terminology simply describes the topology of the diagrams, not the physical phenomena arising from the diagrams. Note that the four-point vertex must be symmetric under the exchange $\mathbf{q}_{1} \leftrightarrow \mathbf{q}_{2}$ and $\mathbf{q}_{1}^{\prime} \leftrightarrow \mathbf{q}_{2}^{\prime}$, so that both "zero sound channels" are characterized by the same parameter $a_{l}$. The parameter $b_{l}$ characterizes the BCS channel.

The exact flow equations for the coupling parameters $a_{l}$ and $b_{l}$ are

$$
\begin{aligned}
& \partial_{l} a_{l}=\left(3-D-2 \eta_{l}\right) a_{l}+\dot{\Gamma}_{l}^{a}, \\
& \partial_{l} b_{l}=\left(3-D-2 \eta_{l}\right) b_{l}+\dot{\Gamma}_{l}^{b},
\end{aligned}
$$

where the coefficients $\dot{\Gamma}_{l}^{a}$ and $\dot{\Gamma}_{l}^{b}$ are defined via the expansion of the function $\dot{\Gamma}_{l}^{(4)}\left(\mathbf{q}_{1}^{\prime}, \mathbf{q}_{2}^{\prime} ; \mathbf{q}_{2}, \mathbf{q}_{1}\right)$ in Eq. (3.15) for small momenta,

$$
\begin{aligned}
\dot{\Gamma}_{l}^{(4)}\left(\mathbf{q}_{1}^{\prime}, \mathbf{q}_{2}^{\prime} ; \mathbf{q}_{2}, \mathbf{q}_{1}\right) & =\dot{\Gamma}_{l}^{(4)}(0,0 ; 0,0) \\
& +\dot{\Gamma}_{l}^{a}\left(\left|\mathbf{q}_{1}-\mathbf{q}_{1}^{\prime}\right|+\left|\mathbf{q}_{1}-\mathbf{q}_{2}^{\prime}\right|\right) \\
& +\dot{\Gamma}_{l}^{b}\left|\mathbf{q}_{1}+\mathbf{q}_{2}\right|+O\left(\mathbf{q}_{i}^{2}\right) .
\end{aligned}
$$

Even if the initial vertex $\tilde{\Gamma}_{l=0}^{(4)}\left(\mathbf{q}_{1}^{\prime}, \mathbf{q}_{2}^{\prime} ; \mathbf{q}_{2}, \mathbf{q}_{1}\right)$ is momentum-independent (corresponding to the initial conditions $a_{0}=b_{0}=0$ ), finite values of these parameters are generated as we iterate the RG. Because in $D=3$ these are marginal, they cannot be ignored and are possibly the source of logarithmic corrections.

Besides $a_{l}$ and $b_{l}$, there is a third marginal parameter in $D=3$, the momentum-independent part of the sixpoint vertex,

$$
v_{l}=\tilde{\Gamma}_{l}^{(6)}(0,0,0 ; 0,0,0),
$$


see Eq. (3.9). It satisfies the exact flow equation

$$
\partial_{l} v_{l}=\left(6-2 D-3 \eta_{l}\right) v_{l}+\dot{\Gamma}_{l}^{(6)}(0,0,0 ; 0,0,0) .
$$

Again, the RG flow generates a finite $v_{l}$ even if initially $v_{0}=0$. At the Wilson-Fisher fixed point in $D=3$ the renormalized $v_{*}=\lim _{l \rightarrow \infty} v_{l}$ is of order unity.

To arrive at the RG equations, we adopt the following approximation scheme: We expand $\dot{\Gamma}_{l}^{(n)}$ to some fixed power in the marginal parameters $a_{l}, b_{l}, v_{l}$, and the relevant parameter $u_{l}$ while keeping higher orders in the relevant parameter $r_{l}$. The truncation is based on the hope that, although $a_{l}, b_{l}, v_{l}$ and $u_{l}$ are not parametrically small, these parameters still remain numerically small. The fixed point values for these parameters which we obtain within this scheme in Sec. VID 1 are indeed much smaller than unity, which gives some a posteriori justification to our approach. To implement the scheme, it is useful to transform Eq. (3.9) into an integral equation,

$$
\begin{aligned}
& \tilde{\Gamma}_{l}^{(n)}\left(\left\{\mathbf{q}_{i}\right\}\right)=e^{[(2-D) n+D] l-n \int_{0}^{l} d \tau \eta_{\tau}} \tilde{\Gamma}_{l=0}^{(n)}\left(\left\{e^{-l} \mathbf{q}_{i}\right\}\right) \\
& \quad+\int_{0}^{l} d t e^{[(2-D) n+D] t-n \int_{l-t}^{l} d \tau \eta_{\tau}} \dot{\Gamma}_{l-t}^{(n)}\left(\left\{e^{-t} \mathbf{q}_{i}\right\}\right)
\end{aligned}
$$

We use these retarded expressions in the calculations of the inhomogeneities $\dot{\Gamma}_{l}^{(m)}$. Since the right hand side in Eq. (6.9) depends on vertices which also have the form (6.9), one quickly arrives at rather complex expressions which we need to truncate. We will only include terms up to second order in $a_{l}, b_{l}, v_{l}$, and $u_{l}$. However, even with this approximation the calculation becomes rather involved. To keep the calculations tractable, we truncate further: To evaluate the flow of parameters associated with the $n$-point vertex, we ignore irrelevant contributions arising from vertices of order $m$ with $m>n+2$. Thus, when we calculate the flow of parameters associated with the $n$-point vertex and encounter a vertex $\tilde{\Gamma}_{l}^{(m)}$ in the calculation with $m>n+2$, we only keep its relevant and marginal part instead of employing Eq. (6.9). Effectively, we neglect in this way infinitely many irrelevant parameters related to higher order vertices. The expectation is, that these irrelevant contributions are numerically small compared to the contribtutions originating from irrelevant terms of lower order vertices. In concrete terms, our truncation is as follows: When we calculate the flow of $v_{l}$, we will consider irrelevant contributions from $\tilde{\Gamma}_{l}^{(8)}$ but ignore contributions from $\tilde{\Gamma}_{l}^{(10)}$ (in this case, there are in fact no second order terms arising from inhomogeneities $\dot{\Gamma}_{l}^{(n)}$ with $n \geq 10$ so that we really have all second order terms included in our flow of $v_{l}$ ). Similarly, to calculate the flow of the four-point vertex parameters $a_{l}, b_{l}$, and $u_{l}$, we ignore contributions arising from $\tilde{\Gamma}_{l}^{(8)}$. This leads to the absence of $v_{l}^{2}$ terms in the RG equations for $a_{l}, b_{l}$, and $u_{l}$, the $\tilde{\Gamma}_{l}^{(8)}$ terms only enter indirectly via the flow of $v_{l}$. To calculate the flow of $r_{l}$ we ignore irrelevant contributions from $\tilde{\Gamma}_{l}^{(n)}$ for $n \geq 6$ which again only enter via their contributions to the flow of $a_{l}$, $b_{l}$, and $u_{l}$.

\section{A. Truncating the flow equation for the six-point vertex}

We begin with the renormalization of $v_{l}$, the momentum independent part of $\tilde{\Gamma}_{l}^{(6)}$, using the flow equations for the six-point vertex from Appendix A. Eq. A2). Note that, working consistently to second order, the last two terms in Eq. (A2), which are at least of third order in the marginal and relevant parameters, should not be included. However, there is a contribution proportional to $v_{l}^{2}$ arising from the eight-point vertex which must be included. To see this, let us explicitly write $\dot{\Gamma}_{l}^{(6)}$ up to second order in the marginal and relevant parameters (and zeroth order in $\eta$ ),

$$
\begin{aligned}
\dot{\Gamma}_{l}^{(6)}\left(\mathbf{q}_{1}^{\prime}, \mathbf{q}_{2}^{\prime}, \mathbf{q}_{3}^{\prime} ; \mathbf{q}_{3}, \mathbf{q}_{2}, \mathbf{q}_{1}\right)=\frac{-3 v_{l}}{1+r_{l}}\left\{3 \mathcal{S}_{(1,2), 3}\left\langle G_{l}\left(\mathbf{q}_{1}+\mathbf{q}_{2}-\hat{\mathbf{q}}\right)\left(u_{l}+a_{l}\left(\left|\mathbf{q}_{1}-\hat{\mathbf{q}}\right|+\left|\mathbf{q}_{2}-\hat{\mathbf{q}}\right|\right)+b_{l}\left|\mathbf{q}_{1}+\mathbf{q}_{2}\right|\right)\right\rangle_{\hat{\mathbf{q}}}\right. \\
\left.+3 \mathcal{S}_{\left(1^{\prime}, 2^{\prime}\right), 3^{\prime}} \mathcal{S}_{(1,2), 3}\left\langle G_{l}\left(\mathbf{q}_{3}-\mathbf{q}_{3}^{\prime}+\hat{\mathbf{q}}\right)\left(u_{l}+a_{l}\left(\left|\mathbf{q}_{3}-\mathbf{q}_{3}^{\prime}\right|\right)+b_{l}\left|\mathbf{q}_{3}+\hat{\mathbf{q}}\right|\right)\right\rangle_{\hat{\mathbf{q}}}+\left(\mathbf{q}_{i} \leftrightarrow \mathbf{q}_{i}^{\prime}\right)\right\} \\
+\frac{1}{1+r_{l}} \int_{0}^{l} d t e^{-(4 D-8) t}\left\langle\dot{\Gamma}_{l-t}^{(8)}\left(e^{-t} \mathbf{q}_{1}^{\prime}, \ldots, e^{-t} \mathbf{q}_{3}^{\prime}, e^{-t} \hat{\mathbf{q}} ; e^{-t} \hat{\mathbf{q}}, e^{-t} \mathbf{q}_{3}, \ldots, e^{-t} \mathbf{q}_{1}\right)\right\rangle_{\hat{\mathbf{q}}}, \quad(6
\end{aligned}
$$

where we used

$$
\begin{aligned}
& \tilde{\Gamma}_{l}^{(8)}\left(\mathbf{q}_{1}^{\prime}, \ldots, \mathbf{q}_{4}^{\prime} ; \mathbf{q}_{4}, \ldots, \mathbf{q}_{1}\right)=\int_{0}^{l} d t e^{-4 \int_{l-t}^{l} d \tau \eta_{\tau}+(8-3 D) t} \\
& \quad \times \dot{\Gamma}_{l-t}^{(8)}\left(e^{-t} \mathbf{q}_{1}^{\prime}, \ldots, e^{-t} \mathbf{q}_{4}^{\prime} ; e^{-t} \mathbf{q}_{4}, \ldots, e^{-t} \mathbf{q}_{1}\right), \quad(6.11)
\end{aligned}
$$

and from Eq. A3 we have

$$
\begin{aligned}
& \dot{\Gamma}_{l}^{(8)}\left(\mathbf{q}_{1}^{\prime}, \ldots, \mathbf{q}_{4}^{\prime} ; \mathbf{q}_{4}, \ldots, \mathbf{q}_{1}\right)= \\
& -2 v_{l}^{2}\left\{16 \mathcal{S}_{(1,2,3), 4} \mathcal{S}_{\left(1^{\prime}, 2^{\prime}, 3^{\prime}\right), 4^{\prime}} \dot{\chi}_{l}\left(\mathbf{q}_{1}^{\prime}+\mathbf{q}_{2}^{\prime}+\mathbf{q}_{3}^{\prime}-\mathbf{q}_{4}\right)\right. \\
& \left.+18 \mathcal{S}_{(1,2),(3,4)} \mathcal{S}_{\left(1^{\prime}, 2^{\prime}\right),\left(3^{\prime}, 4^{\prime}\right)} \dot{\chi}_{l}\left(\mathbf{q}_{1}+\mathbf{q}_{2}-\mathbf{q}_{1}^{\prime}-\mathbf{q}_{2}^{\prime}\right)\right\} \\
& + \text { terms at least cubic in } v_{l}, a_{l}, b_{l}, \text { and } u_{l} .
\end{aligned}
$$


The definition of the symmetrization operators $\mathcal{S}_{(1,2), 3}$ used in Eq. (6.10) and similar ones used in Eq. (6.12) can be found in Eqs. A1bA4a A4b of Appendix Keeping only the zeroth order in a momentum expansion, we have

$$
\begin{aligned}
& \dot{\Gamma}_{l}^{(6)}\left(\left\{\mathbf{q}_{i} \equiv 0\right\}\right)=-12 \beta_{0} v_{l} u_{l}-15 \beta_{0} v_{l} a_{l}-9 \beta_{0} v_{l} b_{l}(6.13) \\
& -\frac{2}{1+r_{l}} \int_{0}^{l} d t e^{-(4 D-8) t} v_{l-t}^{2}\left[16 \dot{\chi}_{l-t}(0)+18 \dot{\chi}_{l-t}\left(e^{-t}\right)\right],
\end{aligned}
$$

where

$$
\beta_{0}=\dot{\chi}_{l}(0)=\frac{1}{\left(1+r_{l}\right)^{2}} .
$$

\section{B. Improved truncation of the flow equation for the four-point vertex}

Including the terms which become marginal in $D=3$ we need to calculate

$$
\begin{aligned}
\dot{\Gamma}_{l}^{(4)}\left(\mathbf{q}_{1}^{\prime}, \mathbf{q}_{2}^{\prime} ; \mathbf{q}_{2}, \mathbf{q}_{1}\right) & \approx \frac{1}{1+r_{l}}\left\langle\tilde{\Gamma}_{l}^{(6)}\left(\mathbf{q}_{1}^{\prime}, \mathbf{q}_{2}^{\prime}, \hat{\mathbf{q}} ; \hat{\mathbf{q}}, \mathbf{q}_{2}, \mathbf{q}_{1}\right)\right\rangle_{\hat{\mathbf{q}}} \\
& +\dot{\Gamma}_{l}^{(4, \mathrm{BCS})}\left(\mathbf{q}_{1}^{\prime}, \mathbf{q}_{2}^{\prime} ; \mathbf{q}_{2}, \mathbf{q}_{1}\right) \\
& +\dot{\Gamma}_{l}^{(4, \mathrm{ZS})}\left(\mathbf{q}_{1}^{\prime}, \mathbf{q}_{2}^{\prime} ; \mathbf{q}_{2}, \mathbf{q}_{1}\right) \\
& +\dot{\Gamma}_{l}^{\left(4, \mathrm{ZS}^{\prime}\right)}\left(\mathbf{q}_{1}^{\prime}, \mathbf{q}_{2}^{\prime} ; \mathbf{q}_{2}, \mathbf{q}_{1}\right),
\end{aligned}
$$

where, instead of Eqs. 4.23 4.24), we now have to calculate the zeroth and first order terms in a momentum expansion of Eq. (6.15) in powers of both the relevant and marginal parameters. We discuss the terms in Eq. 6.15) below, beginning with the contribution from the six-point vertex.

\section{Contributions from $\tilde{\Gamma}_{l}^{(6)}$}

To calculate $\left\langle\tilde{\Gamma}_{l}^{(6)}\left(\mathbf{q}_{1}^{\prime}, \mathbf{q}_{2}^{\prime}, \hat{\mathbf{q}} ; \hat{\mathbf{q}}, \mathbf{q}_{2}, \mathbf{q}_{1}\right)\right\rangle_{\hat{\mathbf{q}}}$, we write, assuming $\tilde{\Gamma}_{l=0}^{(6)}=0$,

$$
\begin{aligned}
& \tilde{\Gamma}_{l}^{(6)}\left(\mathbf{q}_{1}^{\prime}, \mathbf{q}_{2}^{\prime}, \hat{\mathbf{q}} ; \hat{\mathbf{q}}, \mathbf{q}_{2}, \mathbf{q}_{1}\right)=\int_{0}^{l} d t e^{-3 \int_{l-t}^{l} d \tau \eta_{\tau}+(6-2 D) t} \\
& \times \dot{\Gamma}_{l-t}^{(6)}\left(e^{-t} \mathbf{q}_{1}^{\prime}, e^{-t} \mathbf{q}_{2}^{\prime}, e^{-t} \hat{\mathbf{q}} ; e^{-t} \hat{\mathbf{q}}, e^{-t} \mathbf{q}_{2}, e^{-t} \mathbf{q}_{1}\right) .
\end{aligned}
$$

Using Eqs. 6.166.106.12, we can calculate the contribution of $\tilde{\Gamma}_{l}^{(6)}$ to $\dot{\Gamma}_{l}^{(4)}$. Including from the eight-point vertex only the $\mathbf{q}_{i}=0$ contribution and expanding the other contributions up to linear order in the external momenta we have,

$$
\begin{aligned}
\left\langle\dot { \Gamma } _ { l } ^ { ( 6 ) } \left(\lambda \mathbf{q}_{1}^{\prime}, \lambda \mathbf{q}_{2}^{\prime}, \lambda \hat{\mathbf{q}}, \lambda \hat{\mathbf{q}}, \lambda\right.\right. & \left.\left.\mathbf{q}_{2}, \lambda \mathbf{q}_{1}\right)\right\rangle_{\hat{\mathbf{q}}} \approx-\frac{v_{l}}{2}\left\{12 u_{l}\left(\beta_{0}+\dot{\chi}_{l}(\lambda)\right)+a_{l}\left(12 \beta_{0}+8 \dot{\chi}_{l}(\lambda)(1+\lambda)+8 \dot{\Phi}_{l}(\lambda)+2 \dot{\varphi}_{l}(\lambda)\right)\right. \\
& +b_{l}\left(8 \beta_{0}+4 \dot{\chi}_{l}(\lambda)(1+\lambda)+4 \dot{\Phi}_{l}(\lambda)+2 \dot{\varphi}_{l}(\lambda)\right)+\lambda\left|\mathbf{q}_{1}+\mathbf{q}_{2}\right|\left(2 \beta_{1} u_{l}+\left(4 \beta_{1}+2 \beta_{2}\right) a_{l}+2 \beta_{0} b_{l}\right) \\
& \left.+\lambda\left(\left|\mathbf{q}_{1}-\mathbf{q}_{1}^{\prime}\right|+\left|\mathbf{q}_{1}-\mathbf{q}_{2}^{\prime}\right|\right)\left(4 \beta_{1} u_{l}+\left(4 \beta_{0}+4 \beta_{1}+2 \beta_{2}\right) a_{l}+\left(4 \beta_{1}+2 \beta_{2}\right) b_{l}\right)\right\} \\
& -\frac{2}{1+r_{l}} \int_{0}^{l} d t e^{-(4 D-8) t} v_{l-t}^{2}\left[16 \dot{\chi}_{l-t}(0)+18 \dot{\chi}_{l-t}\left(e^{-t}\right)\right]
\end{aligned}
$$

where

$$
\begin{aligned}
& \beta_{1}=\dot{\chi}_{l}^{\prime}(0)=\frac{S_{0}}{\left(1+r_{l}\right)^{2}}-\frac{4 S_{1}}{\left(1+r_{l}\right)^{3}}, \\
& \beta_{2}=\frac{2 S_{1}}{\left(1+r_{l}\right)^{2}}
\end{aligned}
$$

and $S_{0}$ and $S_{1}$ are defined in Eqs. (4.15) and (4.16). We further introduced the functions

$$
\dot{\Phi}_{l}(\lambda)=\frac{2}{1+r_{l}}\left\langle\tilde{G}_{l}\left(\hat{\mathbf{q}}+\hat{\mathbf{q}}^{\prime} \lambda\right)\left|\hat{\mathbf{q}}+\hat{\mathbf{q}}^{\prime} \lambda\right|\right\rangle_{\hat{\mathbf{q}}},
$$

and

$$
\dot{\varphi}_{l}(\lambda)=\frac{1}{\left(1+r_{l}\right)^{2}}\left\langle\left|\hat{\mathbf{q}}+\hat{\mathbf{q}}^{\prime} \lambda\right|\right\rangle_{\hat{\mathbf{q}}} .
$$

Since to zeroth order in $\lambda$ Eq. 6.17) determines the flow of $v_{l}$ via Eq. (6.8), the integral on the right hand side of Eq. (6.16) gives simply $v_{l}$ to order $\lambda^{0}$. We therefore split the angular average of the six-point vertex into two contributions,

$$
\begin{aligned}
& \left\langle\tilde{\Gamma}_{l}^{(6)}\left(\mathbf{q}_{1}^{\prime}, \mathbf{q}_{2}^{\prime}, \hat{\mathbf{q}} ; \hat{\mathbf{q}}, \mathbf{q}_{2}, \mathbf{q}_{1}\right)\right\rangle_{\hat{\mathbf{q}}} \\
& \quad \approx v_{l}+\left\langle\tilde{\Gamma}_{l}^{(6 i)}\left(\mathbf{q}_{1}^{\prime}, \mathbf{q}_{2}^{\prime}, \hat{\mathbf{q}} ; \hat{\mathbf{q}}, \mathbf{q}_{2}, \mathbf{q}_{1}\right)\right\rangle_{\hat{\mathbf{q}}},
\end{aligned}
$$


where $\tilde{\Gamma}_{l}^{(6 i)}$ contains the irrelevant parts of the six-point vertex. To calculate the angular average of the irrelevant part, we can neglect the $\eta_{l}$ dependence of the integral and also approximate the retarded dependence of the param- eters on $l-t$ by an $l$ dependence, since the retardation is exponentially damped. For large $l$ we therefore can write the contributions of the irrelevant part to Eq. (6.16) as follows

$$
\begin{aligned}
\left\langle\tilde{\Gamma}_{l}^{(6 i)}\left(\mathbf{q}_{1}^{\prime}, \mathbf{q}_{2}^{\prime}, \hat{\mathbf{q}} ; \hat{\mathbf{q}}, \mathbf{q}_{2}, \mathbf{q}_{1}\right)\right\rangle_{\hat{\mathbf{q}}} \approx-v_{l} \int_{0}^{1} \frac{d \lambda}{\lambda}\left\{6 u_{l}\left(\dot{\chi}_{l}(\lambda)-\beta_{0}\right)+a_{l}\left(4 \dot{\chi}_{l}(\lambda)(1+\lambda)+4 \dot{\Phi}_{l}(\lambda)-8 \beta_{0}+\left[\dot{\varphi}_{l}(\lambda)-\dot{\varphi}_{l}(0)\right]\right)\right. \\
+b_{l}\left(2 \dot{\chi}_{l}(\lambda)(1+\lambda)+2 \dot{\Phi}_{l}(\lambda)-4 \beta_{0}+\left[\dot{\varphi}_{l}(\lambda)-\dot{\varphi}_{l}(0)\right]\right)+\lambda\left|\mathbf{q}_{1}+\mathbf{q}_{2}\right|\left(\beta_{1} u_{l}+\left(2 \beta_{1}+\beta_{2}\right) a_{l}+\beta_{0} b_{l}\right) \\
\left.+\lambda\left(\left|\mathbf{q}_{1}-\mathbf{q}_{1}^{\prime}\right|+\left|\mathbf{q}_{1}-\mathbf{q}_{2}^{\prime}\right|\right)\left(2 \beta_{1} u_{l}+\left(2 \beta_{0}+2 \beta_{1}+\beta_{2}\right) a_{l}+\left(2 \beta_{1}+\beta_{2}\right) b_{l}\right)\right\}
\end{aligned}
$$

We now turn to the calculation of the BCS, ZS and $\mathrm{ZS}^{\prime}$ diagrams in Eq. 6.15. Keeping only the relevant and marginal terms in the expansion of the four-point vertex, see Eq. (6.3), yields all contributions to second order in the marginal and relevant parameters arising from these diagrams and the irrelevant part of the four-point vertex does not contribute at this order.

\section{Contribution of the $B C S, Z S$ and $Z S^{\prime}$ diagrams}

The contribution from the BCS-channel to Eq. (6.15) is

$$
\begin{aligned}
& \dot{\Gamma}_{l}^{(4, \mathrm{BCS})}\left(\mathbf{q}_{1}^{\prime}, \mathbf{q}_{2}^{\prime} ; \mathbf{q}_{2}, \mathbf{q}_{1}\right) \approx \\
& -\frac{1}{2}\left[u_{l}+b_{l}\left|\mathbf{q}_{1}+\mathbf{q}_{2}\right|\right]^{2} \dot{\chi}_{l}\left(\left|\mathbf{q}_{1}+\mathbf{q}_{2}\right|\right) \\
& -a_{l}\left[u_{l}+b_{l}\left|\mathbf{q}_{1}+\mathbf{q}_{2}\right|\right] \\
& \quad \times \frac{1}{1+r_{l}}\left\langle\frac{\Theta\left(1<\left|\mathbf{q}_{1}+\mathbf{q}_{2}-\hat{\mathbf{q}}\right|<e^{l}\right)}{\left|\mathbf{q}_{1}+\mathbf{q}_{2}-\hat{\mathbf{q}}\right|^{2}+r_{l}}\right. \\
& \left.\quad \times\left[\left|\hat{\mathbf{q}}-\mathbf{q}_{1}^{\prime}\right|+\left|\hat{\mathbf{q}}-\mathbf{q}_{2}^{\prime}\right|+\left|\hat{\mathbf{q}}-\mathbf{q}_{2}\right|+\left|\hat{\mathbf{q}}-\mathbf{q}_{1}\right|\right]\right\rangle_{\hat{\mathbf{q}}} \\
& -\frac{a_{l}^{2}}{1+r_{l}}\left\langle\frac{\Theta\left(1<\left|\mathbf{q}_{1}+\mathbf{q}_{2}-\hat{\mathbf{q}}\right|<e^{l}\right)}{\left.\left|\mathbf{q}_{1}+\mathbf{q}_{2}-\hat{\mathbf{q}}\right|^{2}+r_{l}\right)}\right. \\
& \left.\times\left(\left|\hat{\mathbf{q}}^{\prime}-\mathbf{q}_{1}^{\prime}\right|+\left|\hat{\mathbf{q}}^{\prime}-\mathbf{q}_{2}^{\prime}\right|\right)\left(\left|\hat{\mathbf{q}}-\mathbf{q}_{2}\right|+\left|\hat{\mathbf{q}}-\mathbf{q}_{1}\right|\right)\right\rangle_{\hat{\mathbf{q}}},(6.24)
\end{aligned}
$$

and the contribution from the zero-sound channel is

$$
\begin{aligned}
& \dot{\Gamma}_{l}^{(4, \mathrm{ZS})}\left(\mathbf{q}_{1}^{\prime}, \mathbf{q}_{2}^{\prime} ; \mathbf{q}_{2}, \mathbf{q}_{1}\right) \approx \\
& -\left[u_{l}+a_{l}\left|\mathbf{q}_{1}-\mathbf{q}_{1}^{\prime}\right|\right]^{2} \dot{\chi}_{l}\left(\left|\mathbf{q}_{1}-\mathbf{q}_{1}^{\prime}\right|\right) \\
& -\left(a_{l}+b_{l}\right)\left[u_{l}+a_{l}\left|\mathbf{q}_{1}-\mathbf{q}_{1}^{\prime}\right|\right] \\
& \quad \times \frac{1}{1+r_{l}}\left\langle\frac{\Theta\left(1<\left|\mathbf{q}_{1}-\mathbf{q}_{1}^{\prime}+\hat{\mathbf{q}}\right|<e^{l}\right)}{\left|\mathbf{q}_{1}-\mathbf{q}_{1}^{\prime}+\hat{\mathbf{q}}\right|^{2}+r_{l}}\right. \\
& \left.\quad \times\left[\left|\hat{\mathbf{q}}-\mathbf{q}_{1}^{\prime}\right|+\left|\hat{\mathbf{q}}+\mathbf{q}_{2}^{\prime}\right|+\left|\hat{\mathbf{q}}-\mathbf{q}_{2}\right|+\left|\hat{\mathbf{q}}+\mathbf{q}_{1}\right|\right]\right\rangle_{\hat{\mathbf{q}}} \\
& -\left(a_{l}^{2}+b_{l}^{2}\right) \frac{1}{1+r_{l}}\left\langle\frac{\Theta\left(1<\left|\mathbf{q}_{1}-\mathbf{q}_{1}^{\prime}+\hat{\mathbf{q}}\right|<e^{l}\right)}{\left|\mathbf{q}_{1}-\mathbf{q}_{1}^{\prime}+\hat{\mathbf{q}}\right|^{2}+r_{l}}\right. \\
& \left.\quad \times\left[\left|\hat{\mathbf{q}}-\mathbf{q}_{1}^{\prime} \| \hat{\mathbf{q}}-\mathbf{q}_{2}\right|+\left|\hat{\mathbf{q}}+\mathbf{q}_{1}\right|\left|\hat{\mathbf{q}}+\mathbf{q}_{2}^{\prime}\right|\right]\right\rangle_{\hat{\mathbf{q}}} \\
& -2 a_{l} b_{l} \frac{1}{1+r_{l}}\left\langle\frac{\Theta\left(1<\left|\mathbf{q}_{1}-\mathbf{q}_{1}^{\prime}+\hat{\mathbf{q}}\right|<e^{l}\right)}{\left|\mathbf{q}_{1}-\mathbf{q}_{1}^{\prime}+\hat{\mathbf{q}}\right|^{2}+r_{l}}\right. \\
& \left.\quad \times\left[\left|\hat{\mathbf{q}}-\mathbf{q}_{1}^{\prime} \| \hat{\mathbf{q}}+\mathbf{q}_{2}^{\prime}\right|+\left|\hat{\mathbf{q}}+\mathbf{q}_{1}\right|\left|\hat{\mathbf{q}}-\mathbf{q}_{2}\right|\right]\right\rangle_{\hat{\mathbf{q}}} . \quad(6.25)
\end{aligned}
$$

The contribution from the other zero-sound channel (ZS' in Fig. 1) is obtained by replacing $\mathbf{q}_{1}^{\prime} \leftrightarrow \mathbf{q}_{2}^{\prime}$ on the right-hand side of Eq. (6.25). Expanding to linear order in the momenta, we arrive at

$$
\begin{gathered}
\dot{\Gamma}_{\text {lin }, l}^{(4, \mathrm{BCS})}\left(\mathbf{q}_{1}^{\prime}, \mathbf{q}_{2}^{\prime} ; \mathbf{q}_{2}, \mathbf{q}_{1}\right) \approx-\beta_{0}\left[\frac{u_{l}^{2}}{2}+2 a_{l}^{2}+2 u_{l} a_{l}\right] \\
-\left|\mathbf{q}_{1}+\mathbf{q}_{2}\right|\left[\frac{\beta_{1}}{2} u_{l}^{2}+2\left(\beta_{1}+\beta_{2}\right) a_{l}^{2}+\left(2 \beta_{1}+\beta_{2}\right) u_{l} a_{l}\right. \\
\left.+\beta_{0} u_{l} b_{l}+2 \beta_{0} a_{l} b_{l}\right] .
\end{gathered}
$$

Similarly, we obtain for the contribution from the zerosound-channel,

$$
\begin{gathered}
\dot{\Gamma}_{\operatorname{lin}, l}^{(4, \mathrm{ZS})}\left(\mathbf{q}_{1}^{\prime}, \mathbf{q}_{2}^{\prime} ; \mathbf{q}_{2}, \mathbf{q}_{1}\right) \approx-\beta_{0}\left[u_{l}+a_{l}+b_{l}\right]^{2} \\
-\left|\mathbf{q}_{1}-\mathbf{q}_{1}^{\prime}\right|\left[\beta_{1} u_{l}^{2}+\left(2 \beta_{0}+\beta_{1}+\beta_{2}\right) a_{l}^{2}+\left(\beta_{1}+\beta_{2}\right) b_{l}^{2}\right. \\
+\left(2 \beta_{0}+2 \beta_{1}+\beta_{2}\right) u_{l} a_{l}+\left(2 \beta_{1}+\beta_{2}\right) u_{l} b_{l} \\
\left.+2\left(\beta_{0}+\beta_{1}+\beta_{2}\right) a_{l} b_{l}\right] .
\end{gathered}
$$

The contribution from $\mathrm{ZS}^{\prime}$ is again obtained by replac$\operatorname{ing} \mathbf{q}_{1}^{\prime} \leftrightarrow \mathbf{q}_{2}^{\prime}$. 


\section{Truncated flow equation for the two-point \\ vertex}

To calculate the flow of $r_{l}$, we need to calculate $\dot{\Gamma}_{l}^{(2)}(0)$, see Eq. (3.18). We write it as

$$
\left(1+r_{l}\right) \dot{\Gamma}_{l}^{(2)}(0)=\left\langle\tilde{\Gamma}_{l}^{(4)}(0, \hat{\mathbf{q}}, \hat{\mathbf{q}}, 0)\right\rangle_{\hat{\mathbf{q}}} \approx e^{l-2 \int_{0}^{l} d \tau \eta_{\tau}} u_{0}+\int_{0}^{l} d t e^{t-2 \int_{l-t}^{l} d \tau \eta_{\tau}}\left\langle\dot{\Gamma}_{l-t}^{(4)}\left(0, e^{-t} \hat{\mathbf{q}}, e^{-t} \hat{\mathbf{q}}, 0\right)\right\rangle_{\hat{\mathbf{q}}}
$$

with the second order expression of $\dot{\Gamma}_{l}^{(4)}$ given by Eq. 6.15). Thus, we have

$$
\begin{aligned}
\dot{\Gamma}_{l}^{(4)}(0, \lambda \hat{\mathbf{q}}, \lambda \hat{\mathbf{q}}, 0) \approx & -\frac{1}{2} \dot{\chi}_{l}(\lambda)\left[\left(u_{l}+\lambda b_{l}+a_{l}\right)^{2}+\left(u_{l}+b_{l}+a_{l} \lambda\right)^{2}+\left(u_{l}+a_{l}[1+\lambda]\right)^{2}\right] \\
& -\dot{\phi}_{l}(\lambda)\left[a_{l}\left(u_{l}+b_{l} \lambda+a_{l}\right)+a_{l}\left(u_{l}+b_{l}+a_{l} \lambda\right)+b_{l}\left(u_{l}+a_{l}[1+\lambda]\right)\right] \\
& -\frac{1}{2} \dot{\psi}_{l}(\lambda)\left[2 a_{l}^{2}+b_{l}^{2}\right]-\left(u_{l}+b_{l}+a_{l}\right)\left[\beta_{0} u_{l}+\left(b_{l}+a_{l}\right) \dot{\varphi}_{l}(\lambda)\right] \\
& +\frac{1}{1+r_{l}}\left\langle\tilde{\Gamma}_{l}^{(6)}\left(0, \lambda \hat{\mathbf{q}}, \hat{\mathbf{q}}^{\prime} ; \hat{\mathbf{q}}^{\prime}, \lambda \hat{\mathbf{q}}, 0\right)\right\rangle_{\hat{\mathbf{q}}^{\prime}},
\end{aligned}
$$

with

$$
\dot{\psi}_{l}(\lambda)=\frac{2}{1+r_{l}}\left\langle\tilde{G}_{l}\left(\hat{\mathbf{q}}+\hat{\mathbf{q}}^{\prime} \lambda\right)\left|\hat{\mathbf{q}}+\hat{\mathbf{q}}^{\prime} \lambda\right|^{2}\right\rangle_{\hat{\mathbf{q}}} .
$$

Keeping only terms up to linear order in $\lambda$, which define the flow of $u_{l}, a_{l}$ and $b_{l}$, one finds

$$
\dot{\Gamma}_{l}^{(2)}(0) \approx \frac{u_{l}+a_{l}+b_{l}}{1+r_{l}}
$$

which is just the contribution of the relevant and marginal parts of $\left\langle\tilde{\Gamma}_{l}^{(4)}(0, \hat{\mathbf{q}}, \hat{\mathbf{q}}, 0)\right\rangle_{\hat{\mathbf{q}}}$. Higher orders in $\lambda$ correspond to the contribution of the irrelevant part of the four-point vertex. With

$$
\begin{aligned}
\left\langle\tilde{\Gamma}_{l}^{(4)}(0, \hat{\mathbf{q}}, \hat{\mathbf{q}}, 0)\right\rangle_{\hat{\mathbf{q}}}= & u_{l}+a_{l}+b_{l} \\
& +\left\langle\tilde{\Gamma}_{l}^{(4 \mathrm{i})}(0, \hat{\mathbf{q}}, \hat{\mathbf{q}}, 0)\right\rangle_{\hat{\mathbf{q}}}
\end{aligned}
$$

we can write the contributions of the irrelevant parts (we here ignore the contributions of the irrelevant part of the six-point vertex beyond those which are implicitly contained in the renormalization of $u_{l}$ ) as

$$
\begin{aligned}
\left\langle\tilde{\Gamma}_{l}^{(4 \mathrm{i})}(0,\right. & , \hat{\mathbf{q}}, \hat{\mathbf{q}}, 0)\rangle_{\hat{\mathbf{q}}} \approx \int \frac{d \lambda}{\lambda^{2}}\left\langle\dot{\Gamma}_{l}^{(4, \mathrm{BCS})}(0, \lambda \hat{\mathbf{q}} ; \lambda \hat{\mathbf{q}}, 0)\right. \\
& +\dot{\Gamma}_{l}^{(4, \mathrm{ZS})}(0, \lambda \hat{\mathbf{q}} ; \lambda \hat{\mathbf{q}}, 0)+\dot{\Gamma}_{l}^{\left(4, \mathrm{ZS}^{\prime}\right)}(0, \lambda \hat{\mathbf{q}} ; \lambda \hat{\mathbf{q}}, 0) \\
& -\dot{\Gamma}_{\operatorname{lin}, l}^{(4, \mathrm{BCS})}(0, \lambda \hat{\mathbf{q}} ; \lambda \hat{\mathbf{q}}, 0)-\dot{\Gamma}_{\operatorname{lin}, l}^{(4, \mathrm{ZS})}(0, \lambda \hat{\mathbf{q}} ; \lambda \hat{\mathbf{q}}, 0) \\
& \left.-\dot{\Gamma}_{\operatorname{lin}, l}^{(4, \mathrm{ZS})}(0, \lambda \hat{\mathbf{q}} ; \lambda \hat{\mathbf{q}}, 0)\right\rangle_{\hat{\mathbf{q}}},
\end{aligned}
$$

which is identical to the second and higher order in $\lambda$ contributions contained in Eq. (6.29).

\section{Flow equations of marginal and relevant parameters}

It is now straightforward to write down the flow equations for the marginal and relevant coupling parameters. The flow of $v_{l}$ is determined by Eq. (6.8). With Eq. (6.13) one finds

$$
\begin{aligned}
\partial_{l} v_{l}= & \left(6-2 D-3 \eta_{l}\right) v_{l}-3 \beta_{0} v_{l}\left(4 u_{l}+5 a_{l}+3 b_{l}\right) \\
& -\frac{4 v_{l}^{2}}{1+r_{l}} \int_{0}^{1} d \lambda \lambda^{4 D-9}\left[8 \dot{\chi}_{l}(0)+9 \dot{\chi}_{l}(\lambda)\right],(6.34)
\end{aligned}
$$

where we approximated the retarded dependence on $v_{l-t}$ and $\dot{\chi}_{l-t}$ in Eq. (6.13) by $v_{l}$ and $\dot{\chi}_{l}$ (since the retardation is exponentially damped) and further took for the lower bound of the integral the limit $l \rightarrow \infty$. Eq. 6.34 obviously has a $v_{*}=0$ fixed point solution, which is stable if $3 \beta_{0}\left(4 u_{*}+5 a_{*}+3 b_{*}\right)>6-2 D-3 \eta$.

Collecting all contributions to Eq. (6.15) from Eqs. 6.23 6.26 6.27), and decomposing $\dot{\Gamma}_{l}^{(4)}\left(\mathbf{q}_{1}^{\prime}, \mathbf{q}_{2}^{\prime} ; \mathbf{q}_{2}, \mathbf{q}_{1}\right)$ into a momentum independent contribution and linear contributions according to Eq. (6.6), we find, using Eqs. 3.19 6.4 6.5), the flow equations for the parameters characterizing the 
four-point vertex,

$$
\begin{aligned}
\partial_{l} u_{l}= & \left(4-D-2 \eta_{l}\right) u_{l}-\frac{5}{2} \beta_{0} u_{l}^{2}-\beta_{0} u_{l}\left(6 a_{l}+4 b_{l}\right) \\
-2 \beta_{0}\left(2 a_{l}^{2}+b_{l}^{2}+2 a_{l} b_{l}\right)+\frac{v_{l}}{1+r_{l}}-\frac{v_{l}}{1+r_{l}} \int_{0}^{1} \frac{d \lambda}{\lambda} & \times\left\{6 u_{l}\left(\dot{\chi}_{l}(\lambda)-\beta_{0}\right)+a_{l}\left(4 \dot{\chi}_{l}(\lambda)(1+\lambda)+4 \dot{\Phi}_{l}(\lambda)\right.\right. \\
& \left.-8 \beta_{0}+\left[\dot{\varphi}_{l}(\lambda)-\dot{\varphi}_{l}(0)\right]\right)+b_{l}\left(2 \dot{\chi}_{l}(\lambda)(1+\lambda)\right. \\
& \left.\left.+2 \dot{\Phi}_{l}(\lambda)-4 \beta_{0}+\left[\dot{\varphi}_{l}(\lambda)-\dot{\varphi}_{l}(0)\right]\right)\right\}, \quad(6.35) \\
\partial_{l} a_{l}= & \left(3-D-2 \eta_{l}\right) a_{l}-\beta_{1} u_{l}^{2}-\left(2 \beta_{0}+2 \beta_{1}+\beta_{2}\right) u_{l} a_{l} \\
& -\left(2 \beta_{1}+\beta_{2}\right) u_{l} b_{l}-\left(2 \beta_{0}+\beta_{1}+\beta_{2}\right) a_{l}^{2} \\
& -\left(\beta_{1}+\beta_{2}\right) b_{l}^{2}-2\left(\beta_{0}+\beta_{1}+\beta_{2}\right) a_{l} b_{l} \\
& -\frac{v_{l}}{1+r_{l}}\left\{2 \beta_{1} u_{l}+\left(2 \beta_{0}+2 \beta_{1}+\beta_{2}\right) a_{l}\right. \\
& \left.\quad+\left(2 \beta_{1}+\beta_{2}\right) b_{l}\right\}, \\
\partial_{l} b_{l}= & (3-D-2 \eta) b_{l}-\frac{\beta_{1}}{2} u_{l}^{2}-\left(2 \beta_{1}+\beta_{2}\right) u_{l} a_{l} \\
& -\beta_{0} u_{l} b_{l}-2\left(\beta_{1}+\beta_{2}\right) a_{l}^{2}-2 \beta_{0} a_{l} b_{l} \\
& -\frac{v_{l}}{1+r_{l}}\left\{\beta_{1} u_{l}+\left(2 \beta_{1}+\beta_{2}\right) a_{l}+\beta_{0} b_{l}\right\} .
\end{aligned}
$$

The flow equation for $r_{l}$ is obtained from Eq. (3.18) and Eqs. (6.28 6.33),

$$
\begin{aligned}
\partial_{l} r_{l}= & \left(2-\eta_{l}\right) r_{l}+\frac{u_{l}+a_{l}+b_{l}}{1+r_{l}} \\
& +\frac{1}{1+r_{l}}\left\langle\tilde{\Gamma}_{l}^{(4 \mathrm{i})}(0, \hat{\mathbf{q}} ; \hat{\mathbf{q}}, 0)\right\rangle_{\hat{\mathbf{q}}} .
\end{aligned}
$$

Finally, we need to determine the flowing anomalous dimension $\eta_{l}$ before we can analyse the fixed point of the RG equations. For now, we keep only the marginal and relevant part of the four-point vertex in Eq. (3.21) and arrive at

$$
\eta_{l} \approx \beta_{3}\left(a_{l}+b_{l}\right)
$$

where

$$
\beta_{3}=\left.\frac{1}{1+r_{l}} \frac{\partial}{\partial q^{2}}\left\langle\left|\hat{\mathbf{q}}^{\prime}+\mathbf{q}\right|\right\rangle_{\hat{\mathbf{q}}^{\prime}}\right|_{q^{2}=0}=\frac{D-1}{2 D\left(1+r_{l}\right)} .
$$

\section{Fixed point values in $D=3$}

In $D=3$, we have

$$
\begin{aligned}
& \dot{\Phi}_{l}(\lambda)=\frac{\lambda+\sqrt{-r_{l}} \operatorname{arctanh}\left[\frac{\lambda \sqrt{-r_{l}}}{1+r_{l}+\lambda}\right]}{\lambda\left(1+r_{l}\right)}, \\
& \dot{\psi}_{l}(\lambda)=\frac{\lambda(2+\lambda)+r_{l} \ln \left[\frac{1+r_{l}}{(1+\lambda)^{2}+r_{l}}\right]}{2 \lambda\left(1+r_{l}\right)}, \\
& \dot{\varphi}_{l}(\lambda)=\frac{3+\lambda^{2}}{3\left(1+r_{l}\right)^{2}}
\end{aligned}
$$

and

$$
\begin{aligned}
& \beta_{0}=\frac{1}{\left(1+r_{l}\right)^{2}}, \\
& \beta_{1}=\frac{r_{l}-1}{2\left(1+r_{l}\right)^{3}}, \\
& \beta_{2}=\frac{1}{2\left(1+r_{l}\right)^{2}}, \\
& \beta_{3}=\frac{1}{3\left(1+r_{l}\right)} .
\end{aligned}
$$

Setting the left-hand sides of the five flow equations (6.346.356.366.376.38) for the two relevant coupling parameters $r_{l}$ and $u_{l}$ and the three marginal coupling parameters $a_{l}, b_{l}$ and $v_{l}$ equal to zero and employing Eq. 6.39) for the anomalous dimension, we obtain numerically the fixed point values

$$
\begin{array}{ll}
r_{*} \approx-0.0996, & u_{*} \approx 0.122, \quad a_{*} \approx 0.0371, \\
b_{*} \approx 0.0339, & v_{*}=0, \quad \eta \approx 0.0263 .
\end{array}
$$

The value of $\eta$ is now much closer to the correct value $\eta \approx 0.038$ and the inclusion of the marginal terms certainly improves upon the analysis including only $u_{l}$. However, the fact that $v_{*}=0$ is an artifact of the approximation which ignores all terms of third or higher order in the relevant and marginal parameters. A simple improvement can be obtained by including from the third order terms in the renormalization of $v_{l}$ the marginal contributions. In that case, only the flow of $v_{l}$ is modified and Eq. 6.34) becomes

$$
\begin{gathered}
\partial_{l} v_{l}=\left(6-2 D-3 \eta_{l}\right) v_{l}-3 \beta_{0} v_{l}\left(4 u_{l}+5 a_{l}+3 b_{l}\right) \\
-\frac{4 v_{l}^{2}}{1+r_{l}} \int_{0}^{1} d \lambda \lambda^{4 D-9}\left[8 \dot{\chi}_{l}(0)+9 \dot{\chi}_{l}(\lambda)\right] \\
+\frac{\left(u_{l}+a_{l}+b_{l}\right)}{\left(1+r_{l}\right)^{3}}\left[12\left(u_{l}+a_{l}+b_{l}\right)^{2}\right. \\
\left.+9\left(u_{l}+2 a_{l}\right)^{2}\right]
\end{gathered}
$$

The resulting fixed point values with the 3rd order terms are:

$$
\begin{aligned}
& r_{*} \approx-0.134, \quad u_{*} \approx 0.127, \quad a_{*} \approx 0.0693, \\
& b_{*} \approx 0.0639, \quad v_{*} \approx 0.0917, \quad \eta \approx 0.0513 .
\end{aligned}
$$

The value for $\eta$ is now slightly too large and a quick convergence is not obtained. However, one may expect from this result that a consistent treatment to third order, a very complex calculation, would indeed improve upon the second order result. For completeness, let us also mention the results of another possible approximation, where one keeps only the marginal and relevant terms of the vertices entering the $\dot{\Gamma}_{l}^{(n)}$ expressions. Within such an approximation, the flow equations for $a_{l}$ and $b_{l}$ remain identical to Eqs. 6.366.37) whereas the flow equations 
for $u_{l}, v_{l}$ and $r_{l}$ would simplify to

$$
\begin{aligned}
\partial_{l} v_{l}= & \left(6-2 D-3 \eta_{l}\right) v_{l}-3 \beta_{0} v_{l}\left(4 u_{l}+5 a_{l}+3 b_{l}\right) \\
+ & \frac{\left(u_{l}+a_{l}+b_{l}\right)}{\left(1+r_{l}\right)^{3}}\left[12\left(u_{l}+a_{l}+b_{l}\right)^{2}\right. \\
& \left.+9\left(u_{l}+2 a_{l}\right)^{2}\right] \\
\partial_{l} u_{l}= & \left(4-D-2 \eta_{l}\right) u_{l}-\frac{5}{2} \beta_{0} u_{l}^{2}-\beta_{0} u_{l}\left(6 a_{l}+4 b_{l}\right) \\
- & 2 \beta_{0}\left(2 a_{l}^{2}+b_{l}^{2}+2 a_{l} b_{l}\right)+\frac{v_{l}}{1+r_{l}} \\
\partial_{l} r_{l}= & \left(2-\eta_{l}\right) r_{l}+\frac{u_{l}+a_{l}+b_{l}}{1+r_{l}} .
\end{aligned}
$$

The corresponding fixed point values are

$$
\begin{aligned}
& r_{*} \approx-0.227, \quad u_{*} \approx 0.178, \quad a_{*} \approx 0.0838, \\
& b_{*} \approx 0.0767, \quad v_{*} \approx 0.255, \quad \eta \approx 0.0692 .
\end{aligned}
$$

The value for $\eta$ is worse in this approximation compared to those which also include irrelevant parts of the vertices. Problems with a similar implementation of the sharp cutoff formulation were previously reported in Ref. [26]. Ignoring the third order terms in the flow of $v_{l}$, Eq. [6.48), leads to fixed point values almost identical to those listed in Eqs. 6.45).

\section{Including irrelevant terms in the equation for $\eta$}

Eq. (6.39) for the flowing anomalous dimension includes only the marginal parts of the four-point vertex. To include also irrelevant contributions we need to evaluate the irrelevant parts of $\dot{\Gamma}_{l}^{(4)}$, i. e. we must calculate both the irrelevant contribution from the six-point vertex and the $\mathrm{BCS}, \mathrm{ZS}$ and $\mathrm{ZS}^{\prime}$ contributions to

$$
\begin{aligned}
& \eta=\frac{1}{1+r_{*}} \int_{0}^{1} d \lambda \lambda^{-2+2 \eta} \\
& \times\left.\frac{\partial}{\partial q^{2}}\left\langle\dot{\Gamma}_{\infty}^{(4)}\left(\lambda \mathbf{q}, \lambda \hat{\mathbf{q}}^{\prime}, \lambda \hat{\mathbf{q}}^{\prime}, \lambda \mathbf{q}\right)\right\rangle_{\hat{\mathbf{q}}^{\prime}}\right|_{q^{2}=0}
\end{aligned}
$$

using Eqs. 6.106.166.236.246.25). This is a somewhat lengthy calculation, and we refer to Appendix $\mathbb{B}$ for a table of the required integrals. The resulting values of $\eta$ are generally very small, in the approximation with all terms up to second order in the relevant and marginal parameters we obtain $\eta \approx 0.0127$. Including the third order terms in the flow of $v_{l}$ we get the even smaller value $\eta \approx 0.00797$. It is not completely clear why the results for $\eta$ become worse on including irrelevant terms in the calculation for $\eta_{l}$. A possible problem is that in the calculation of $\eta$, Eq. (6.52), irrelevant terms contribute which however do not also enter the renormalization of $u_{l}, a_{l}$ and $b_{l}$. Terms similar to the irrelevant ones contained in Eq. 66.52) would only enter the renormalization of other parameters at third order in the marginal and relevant parameters. This might suggest that one should use the same level of approximation for the vertex $\tilde{\Gamma}_{l}^{(4)}$ in calculating $\eta$ and $\dot{\Gamma}_{l}^{(4)}$ for the flow equations for $u_{l}$, $a_{l}$ and $b_{l}$. In that case, the first order expression for $\eta$, Eq. 6.39), should be used together with the second order expressions for the flows of $u_{l}, a_{l}$ and $b_{l}$. As shown in the previous subsection, this indeed leads to better approximations of $\eta$.

\section{CALCULATION OF $\sigma(x)$ INCLUDING ONLY MARGINAL PARAMETERS}

We demonstrate here the importance of the irrelevant parts of the four-point vertex for a correct description of the large wave vector regime and show that a treatment that ignores irrelevant terms leads to an incorrect description of $\sigma(x)$ in the regime $x \gg 1$ and the wrong result $\Delta T_{c} \propto u_{0} \ln u_{0}$. Ignoring in Eq. (6.3) the irrelevant contributions, one obtains from Eq. (3.12) the following expression for the subtracted function $\dot{\Gamma}_{l}^{(2 m)}(\mathbf{q})=$ $\dot{\Gamma}_{l}^{(2)}(\mathbf{q})-\dot{\Gamma}_{l}^{(2)}(0)$ (the superscript $m$ indicates that only marginal terms remain)

$$
\begin{aligned}
\dot{\Gamma}_{l}^{(2 m)}(\mathbf{q}) & \approx \frac{a_{l}+b_{l}}{1+r_{l}}\left(\left\langle\left|\hat{\mathbf{q}}^{\prime}+\mathbf{q}\right|\right\rangle_{\hat{\mathbf{q}}^{\prime}}-1\right) \\
& =\frac{a_{l}+b_{l}}{1+r_{l}} \times\left\{\begin{array}{cc}
\frac{q^{2}}{3} & \text { for } q<1, \\
\frac{3 q^{2}-3 q+1}{3 q} & \text { for } q>1 .
\end{array}\right.
\end{aligned}
$$

Via Eq. (4.49) we obtain the scaling function $\sigma(x)$ (we here restrict the discussion to $D=3$ ),

$$
\begin{aligned}
\sigma(x) \approx & x^{2} \int_{0}^{l_{c}-\ln x} d l e^{-2\left(l-l_{c}\right)+\int_{0}^{l} d \tau \eta_{\tau}} \frac{a_{l}+b_{l}}{3\left(1+r_{l}\right)} \\
& +\int_{l_{c}-\ln x}^{\infty} d l e^{\int_{0}^{l} d \tau \eta_{\tau}} \frac{a_{l}+b_{l}}{3\left(1+r_{l}\right)} \\
& \times\left[e^{-\left(l-l_{c}\right)} x-e^{-2\left(l-l_{c}\right)}+\frac{1}{3 x} e^{-3\left(l-l_{c}\right)}\right] .
\end{aligned}
$$

This equation is valid for $\ln x<l_{c}$, for $\ln x \geq l_{c}$ the integral bound $l_{c}-\ln x$ is replaced by zero, i. e. the first integral vanishes. Since in $D=3$ we have $\left(a_{l}+b_{l}\right) /(3[1+$ $\left.\left.r_{l}\right]\right) \approx \eta_{l}$ (see Eq. (6.39) $), \sigma(x)$ is determined by the flow of $\eta_{l}$ alone in this approximation. Let us examine first the case $x \gg 1$. The first integral then extends only over small $l \ll l_{c}$. In that case, all marginal parameters are small and we may ignore the contribution of the integral of $\eta_{\tau}$ in the exponent and approximate the flow of the marginal parameters as $a_{l}, b_{l} \propto e^{2\left(l-l_{c}\right)} u_{*}^{2}$ since $\partial_{l} a_{l} \approx$ $2 \partial_{l} b_{l} \approx u_{l}^{2} \propto e^{2\left(l-l_{c}\right)} u_{*}^{2}$ for $l \ll l_{c}$. The contribution of the first integral in Eq. (77.2) therefore vanishes like $x^{2}\left(l_{c}-\ln x\right) u_{*}^{2}$ as $x$ approaches the UV cutoff, $x \rightarrow e^{l_{c}}$ and makes no contribution for $x>e^{l_{c}}$. To investigate the contribution of the second integral, we split it into two parts,

$$
\int_{l_{c}-\ln x}^{\infty}=\int_{l_{c}-\ln x}^{l_{c}}+\int_{l_{c}}^{\infty}
$$


where, to estimate the contribution of the regime $l<l_{c}$, we use the same approximation as for the first integral in Eq. (7.2). This yields a contribution $\propto(x-1-\ln x-$ $(1-x) / 3 x)$. To estimate the contribution of the integral for $l>l_{c}$ we may replace the parameters $a_{l}, b_{l}, r_{l}$ and $\eta_{l}$ by their fixed point values and obtain a contribution $\propto \eta x /(1-\eta)-\eta /(2-\eta)+\eta /(3 x(3-\eta))$. We have employed Eq. (6.39) to arrive at this result. Hence, the dominant behavior of $\sigma(x)$ for large $x$ is linear in $x$. This large $x$ behavior prohibits the removal of the UV cutoff in the integral determining the temperature shift, Eq. (5.1), and one thus would incorrectly predict from Eq. (5.1) a term for the $T_{c}$ shift behaving like $u_{0} \ln u_{0}$ in $D=3$. Thus, inclusion of the irrelevant terms is essential for determining correctly the shift of the critical temperature. The absence of irrelevant terms in the Wilson RG analysis carried out by Bijlsma and Stoof [9] seems to be responsible for the $u_{0} \ln u_{0}$ behavior they find for the $T_{c}$-shift.

On the other hand, an approach which explicitly includes marginal terms but ignores irrelevant ones is well suited to describe the scaling regime $x \rightarrow 0$. In this case, the dominant contributions to Eq. (7.2) come from $l>l_{c}$ where we replace the coupling constants by their fixed point values and obtain $\sigma(x) \approx A_{3} x^{2-\eta}$ with

$$
A_{3}=1+\frac{3 \eta}{1-\eta}-\frac{3 \eta}{2-\eta}+\frac{\eta}{3-\eta} \approx 1+\frac{11}{6} \eta .
$$

This expression is in relatively good agreement with our result from Sec. IVD2 Eq. 4.57), where we obtained $A_{3} \approx 1.17$ for $\eta \approx 0.104$, whereas Eq. (7.4) would predict $A_{3} \approx 1.22$ for the same $\eta$. Furthermore, for the generally accepted value $\eta \approx 0.038$ Eq. (7.4) predicts $A_{3} \approx 1.07$, which compares relatively well with a recent Monte-Carlo result [4] $A_{3} \approx 1.04$.

\section{CONCLUSION}

In this work we have shown how the functional RG can be employed to calculate the complete scaling function of the zero frequency self-energy of weakly interacting bosons in $3 \leq D<4$. The scaling function describes the cross-over from the small wave vector regime with anomalous scaling to the large wave vector regime where logarithmic divergences appear in $D=3$. A simple truncation of the flow equations at the four-point vertex which ignores the six-point vertex and does not treat the marginal parts of the four-point vertex consistently leads nonetheless to a very accurate description of both the cross-over regime and the large wave vector regime while giving satisfying results for the anomalous scaling regime even in $D=3$. We have used this scaling function to calculate the interaction induced shift of the critical temperature and obtained $\Delta T_{c} / T_{c}=1.23 \mathrm{an}^{1 / 3}$, a result which compares very well with those obtained within the variational perturbation theory [16, 17] and Monte-Carlo simulations [14, 15]. The technique and truncation scheme employed here might also prove useful to obtain energy and/or momentum-dependent scaling functions in other critical systems. A similar truncation has already been applied to calculate the self-energy in the vicinity of the Luttinger liquid fixed point of fermions in one dimension 27].

We have further investigated the fixed point structure using several approximation schemes which include the marginal terms associated with the four-point and sixpoint vertices in $D=3$. These schemes generally lead to an improved anomalous dimension, however, we did not get a quick convergence of $\eta$ to the accepted value. Nonetheless, the best value for $\eta$ which we obtain, $\eta \approx$ 0.0513 is rather close to the one obtained from the first order average action approximation, $\eta \approx 0.049[28]$ and it is feasible that an improved treatment, e. g. a consistent treatment to third order in the marginal and relevant parameters, would indeed produce quite accurate results. Unfortunately, such a calculation is rather lengthy and seems not to be an efficient way of calculating accurate values of critical exponents.

Finally, we have analysed the two-point scaling function within an approach which includes marginal and ignores irrelevant terms of the four-point function. It was shown, that if one ignores the irrelevant terms contained in the higher-order momentum dependence of the fourpoint vertex, one obtains an incorrect UV behavior of the scaling function and hence the wrong functional dependence $\Delta T_{c} / T_{c} \propto a n^{1 / 3} \ln \left(a n^{1 / 3}\right)$ of the critical temperature shift in $D=3$. However, the approach worked well in the critical regime where we used it to calculate the prefactor of the anomalous scaling term, which was shown to be in good agreement with numerical results [4]. While we have not attempted to calculate the scaling function including both marginal and irrelevant terms, it seems certainly feasible to do so within an approximation which ignores the six-point vertex (since $v_{*}=0$ in a calculation to second order in $u_{l}, a_{l}, b_{l}$ and $v_{l}$, this would be consistent). The fixed point value of $\eta$ and hence the description of the small wave vector regime improves within such an approach. However, we do not expect that the inclusion of marginal terms would have much effect on the crossover regime $x \approx 1$, since this regime seems to be well described already in the simpler truncation used in Sec. IV] as is evident from the rather accurate value for the $T_{c}$-shift.

\section{APPENDIX A: FLOW EQUATIONS FOR THE SIX- AND EIGHT-POINT VERTEX}

Below, we discuss the flow equations of the six- and eight-point vertex for the unrescaled vertex functions. The inhomogeneous part of the flow equations for the classical rescaled flow equations follow via the replacement rule $\dot{G}_{\Lambda}(K) \rightarrow-\dot{G}_{l}(\mathbf{q}), G_{\Lambda}(K) \rightarrow-\tilde{G}_{l}(\mathbf{q})$, $\Gamma_{\Lambda}^{(2 n)} \rightarrow \tilde{\Gamma}_{l}^{(2 n)}, \int_{K} \rightarrow \int_{\mathbf{q}}$, and multiplying the resulting expression by an overall minus sign. 


\section{Six-point vertex}

For the calculation of the critical exponents in $D=$ 3 we need the six-point vertex, see Sec. VI We first define the symmetrization operators $\mathcal{S}_{1,2,3}$ and $\mathcal{S}_{1,(2,3)}$ as follows,

$$
\begin{gathered}
\mathcal{S}_{1,2,3} f(1,2,3)=\frac{1}{6}[f(1,2,3)+f(2,3,1)+f(3,1,2) \\
+f(3,2,1)+f(2,1,3)+f(1,3,2)], \quad(\mathrm{A} 1 \mathrm{a}) \\
\mathcal{S}_{1,(2,3)} f(1,2,3)=\mathcal{S}_{(2,3), 1} f(1,2,3) \\
\left.=\frac{1}{3}[f(1,2,3)+f(2,1,3)+f(3,2,1)\} \mathrm{A} 1 \mathrm{~b}\right)
\end{gathered}
$$

Given a function $f(1,2,3)$ that is already symmetric with respect to the pair $(2,3)$, the function $\mathcal{S}_{1,(2,3)} f(1,2,3)$ is a totally symmetric function. The flow equation of the six-point vertex is given by [13] (the equation is shown graphically in Fig. 6)

$$
\begin{aligned}
& \partial_{\Lambda} \Gamma_{\Lambda}^{(6)}\left(K_{1}^{\prime}, K_{2}^{\prime}, K_{3}^{\prime} ; K_{3}, K_{2}, K_{1}\right)=\int_{K} \dot{G}_{\Lambda}(K) \Gamma_{\Lambda}^{(8)}\left(K_{1}^{\prime}, K_{2}^{\prime}, K_{3}^{\prime}, K ; K, K_{3}, K_{2}, K_{1}\right) \\
& +3 \int_{K}\left\{\mathcal{S}_{3,(2,1)}\left[\dot{G}_{\Lambda}(K) G_{\Lambda}\left(K^{\prime}\right) \Gamma_{\Lambda}^{(6)}\left(K_{1}^{\prime}, K_{2}^{\prime}, K_{3}^{\prime} ; K_{3}, K^{\prime}, K\right) \Gamma_{\Lambda}^{(4)}\left(K, K^{\prime} ; K_{2}, K_{1}\right)\right]_{K^{\prime}=K_{1}+K_{2}-K}\right. \\
& \left.\quad+\mathcal{S}_{\left(1^{\prime}, 2^{\prime}\right), 3^{\prime}}\left[\dot{G}_{\Lambda}(K) G_{\Lambda}\left(K^{\prime}\right) \Gamma_{\Lambda}^{(4)}\left(K_{1}^{\prime}, K_{2}^{\prime} ; K^{\prime}, K\right) \Gamma_{\Lambda}^{(6)}\left(K, K^{\prime}, K_{3}^{\prime} ; K_{3}, K_{2}, K_{1}\right)\right]_{K^{\prime}=K_{1}^{\prime}+K_{2}^{\prime}-K}\right\} \\
& +9 \int_{K} \mathcal{S}_{\left(1^{\prime}, 2^{\prime}\right), 3^{\prime}} \mathcal{S}_{3,(2,1)}\left[\left[\dot{G}_{\Lambda}(K) G_{\Lambda}\left(K^{\prime}\right)+G_{\Lambda}(K) \dot{G}_{\Lambda}\left(K^{\prime}\right)\right] \Gamma_{\Lambda}^{(4)}\left(K_{3}^{\prime}, K^{\prime} ; K, K_{3}\right) \Gamma_{\Lambda}^{(6)}\left(K_{1}^{\prime}, K_{2}^{\prime}, K ; K^{\prime}, K_{2}, K_{1}\right)\right]_{K^{\prime}=K_{3}-K_{3}^{\prime}+K} \\
& +9 \int_{K} \mathcal{S}_{\left(1^{\prime}, 2^{\prime}\right), 3^{\prime}} \mathcal{S}_{3,(2,1)}\left[\left[\dot{G}_{\Lambda}(K) G_{\Lambda}\left(K^{\prime}\right) G_{\Lambda}\left(K^{\prime \prime}\right)+G_{\Lambda}(K) \dot{G}_{\Lambda}\left(K^{\prime}\right) G_{\Lambda}\left(K^{\prime \prime}\right)+G_{\Lambda}(K) G_{\Lambda}\left(K^{\prime}\right) \dot{G}_{\Lambda}\left(K^{\prime \prime}\right)\right]\right. \\
& \left.\quad \times \Gamma_{\Lambda}^{(4)}\left(K_{1}^{\prime}, K_{2}^{\prime} ; K, K^{\prime}\right) \Gamma_{\Lambda}^{(4)}\left(K_{3}^{\prime}, K^{\prime} ; K^{\prime \prime}, K_{3}\right) \Gamma_{\Lambda}^{(4)}\left(K^{\prime \prime}, K ; K_{2}, K_{1}\right)\right]_{K^{\prime}=K_{1}^{\prime}+K_{2}^{\prime}-K}^{K^{\prime \prime}=K_{1}+K_{2}-K} \\
& +36 \int_{K} \mathcal{S}_{1^{\prime}, 2^{\prime}, 3^{\prime}} \mathcal{S}_{1,2,3}\left[\dot{G}_{\Lambda}(K) G_{\Lambda}\left(K^{\prime}\right) G_{\Lambda}\left(K^{\prime \prime}\right) \Gamma_{\Lambda}^{(4)}\left(K_{1}^{\prime}, K^{\prime} ; K, K_{1}\right) \Gamma_{\Lambda}^{(4)}\left(K_{2}^{\prime}, K^{\prime \prime} ; K^{\prime}, K_{2}\right) \Gamma_{\Lambda}^{(4)}\left(K_{3}^{\prime}, K ; K^{\prime \prime}, K_{3}\right)\right]_{K^{\prime}=K_{1}-K_{1}^{\prime}+K}^{K^{\prime \prime}=K_{3}^{\prime}-K_{3}+K}
\end{aligned}
$$

\section{Eight-point vertex}

In Eq. (6.10) we also need the following terms from the inhomogeneity of the eight-point vertex,

$$
\begin{aligned}
& \partial_{\Lambda} \Gamma_{\Lambda}^{(8)}\left(K_{1}^{\prime}, K_{2}^{\prime}, K_{3}^{\prime}, K_{4}^{\prime} ; K_{4}, K_{3}, K_{2}, K_{1}\right) \\
& =16 \int_{K} \mathcal{S}_{(1,2,3), 4} \mathcal{S}_{\left(1^{\prime}, 2^{\prime}, 3^{\prime}\right), 4^{\prime}}\left[\dot{G}_{\Lambda}(K) G_{\Lambda}\left(K^{\prime}\right) \Gamma_{\Lambda}^{(6)}\left(K_{1}^{\prime}, K_{2}^{\prime}, K_{3}^{\prime} ; K_{4}, K^{\prime}, K\right) \Gamma_{\Lambda}^{(6)}\left(K, K^{\prime}, K_{4}^{\prime} ; K_{3}, K_{2}, K_{1}\right)\right]_{K^{\prime}=K_{1}^{\prime}+K_{2}^{\prime}+K_{3}^{\prime}-K_{4}-K} \\
& \quad+9 \int_{K} \mathcal{S}_{\left(1^{\prime}, 2^{\prime}\right),\left(3^{\prime}, 4^{\prime}\right)} \mathcal{S}_{(1,2),(3,4)}\left[\left[\dot{G}_{\Lambda}(K) G_{\Lambda}\left(K^{\prime}\right)+G_{\Lambda}(K) \dot{G}_{\Lambda}\left(K^{\prime}\right)\right]\right. \\
& \left.\quad \times \Gamma_{\Lambda}^{(6)}\left(K_{3}^{\prime}, K_{4}^{\prime}, K^{\prime} ; K, K_{4}, K_{3}\right) \Gamma_{\Lambda}^{(6)}\left(K_{1}^{\prime}, K_{2}^{\prime}, K ; K^{\prime}, K_{2}, K_{1}\right)\right]_{K^{\prime}=K_{1}^{\prime}+K_{2}^{\prime}-K_{1}-K_{2}+K}+\ldots,
\end{aligned}
$$

with

$$
\begin{array}{r}
\mathcal{S}_{(1,2,3), 4} f(1,2,3,4)=\frac{1}{4}[f(1,2,3,4)+f(4,2,3,1) \\
+f(1,4,3,2)+f(1,2,4,3)], \quad(\mathrm{A} 4 \mathrm{a})
\end{array}
$$

$$
\begin{aligned}
\mathcal{S}_{(1,2),(3,4)} f(1,2,3,4)=\frac{1}{3}[ & f(1,2,3,4)+f(1,3,2,4) \\
& +f(1,4,2,3)] .
\end{aligned}
$$




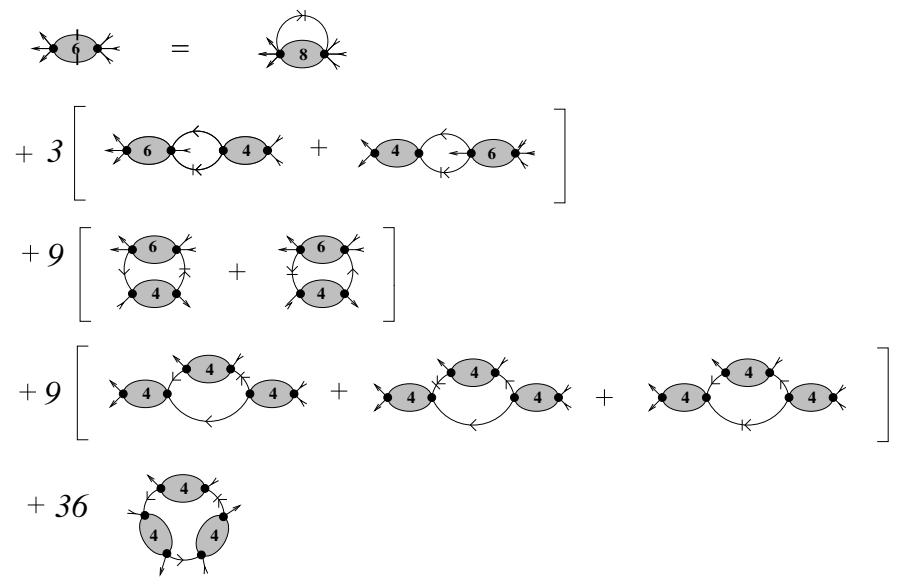

FIG. 6: Diagrammatic representation of the flow equation for the six-point vertex, see Eq. (A2).

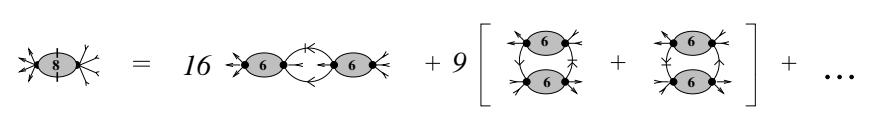

FIG. 7: Diagrammatic representation of the terms of the flow equation for the eight-point vertex which are of order $v_{l}^{2}$, see Eq. A3 . The omitted terms are at least cubic in the parameters $a_{l}, b_{l}, v_{l}$ and $u_{l}$.

\section{APPENDIX B: SOME INTEGRALS ENTERING THE CONTRIBUTION FROM IRRELEVANT TERMS TO $\eta$}

We here calculate the irrelevant contributions to the flow of $\eta$, using Eq. (6.52) of Sec. VID2 Let us define

$$
\begin{aligned}
\dot{f}_{l}\left(\mathbf{q}_{1}, \mathbf{q}_{2}\right)= & \frac{1}{1+r_{l}}\left\langle\frac{\Theta\left(1<\left|\mathbf{q}_{1}+\mathbf{q}_{2}-\hat{\mathbf{q}}\right|<e^{l}\right)}{\left|\mathbf{q}_{1}+\mathbf{q}_{2}-\hat{\mathbf{q}}\right|^{2}+r_{l}}\right. \\
& \left.\times\left[\left|\hat{\mathbf{q}}-\mathbf{q}_{1}\right|+\left|\hat{\mathbf{q}}-\mathbf{q}_{2}\right|\right]\right\rangle_{\hat{\mathbf{q}}}, \\
\dot{g}_{l}\left(\mathbf{q}_{1}, \mathbf{q}_{2}\right)= & \frac{1}{1+r_{l}}\left\langle\frac{\Theta\left(1<\left|\mathbf{q}_{1}+\mathbf{q}_{2}-\hat{\mathbf{q}}\right|<e^{l}\right)}{\left|\mathbf{q}_{1}+\mathbf{q}_{2}-\hat{\mathbf{q}}\right|^{2}+r_{l}}\right. \\
& \left.\times\left[\left|\hat{\mathbf{q}}-\mathbf{q}_{1}\right|^{2}+\left|\hat{\mathbf{q}}-\mathbf{q}_{2}\right|^{2}\right]\right\rangle \hat{\mathbf{q}}, \\
\dot{h}_{l}\left(\mathbf{q}_{1}, \mathbf{q}_{2}\right)= & \frac{2}{1+r_{l}}\left\langle\frac{\Theta\left(1<\left|\mathbf{q}_{1}+\mathbf{q}_{2}-\hat{\mathbf{q}}\right|<e^{l}\right)}{\left|\mathbf{q}_{1}+\mathbf{q}_{2}-\hat{\mathbf{q}}\right|^{2}+r_{l}}\right. \\
& \left.\times\left|\hat{\mathbf{q}}-\mathbf{q}_{1}\right|\left|\hat{\mathbf{q}}-\mathbf{q}_{2}\right|\right\rangle_{\hat{\mathbf{q}}} .
\end{aligned}
$$

To calculate the contributions of the six-point vertex to Eq. 6.52), we need to evaluate (we restrict the results to $D=3$ )

$$
\begin{aligned}
\left.\frac{\partial}{\partial q^{2}}\left\langle\dot{\Gamma}_{l}^{6}\left(\lambda \mathbf{q}, \lambda \hat{\mathbf{q}}^{\prime}, \lambda^{\prime} \hat{\mathbf{q}}^{\prime \prime} ; \lambda^{\prime} \hat{\mathbf{q}}^{\prime \prime}, \lambda \hat{\mathbf{q}}^{\prime}, \lambda \mathbf{q}\right)\right\rangle_{\hat{\mathbf{q}}^{\prime}, \hat{\mathbf{q}}^{\prime \prime}}\right|_{\mathbf{q}^{2}=0}= \\
\quad-v_{l}\left\{3 u_{l}\left[\dot{\chi}_{l}^{\prime \prime}(\lambda, \lambda)+\dot{\chi}_{l}^{\prime \prime}\left(\lambda, \lambda^{\prime}\right)\right]+2\left(b_{l}+2 a_{l}\right)\left[\dot{f}_{l}^{\prime \prime}(\lambda, \lambda)+\dot{f}_{l}^{\prime \prime}\left(\lambda, \lambda^{\prime}\right)+\dot{\alpha}_{l}\left(\lambda, \lambda^{\prime}\right)\right]+\left(b_{l}+a_{l}\right) \dot{\varphi}_{l}^{\prime \prime}(\lambda)\right\},
\end{aligned}
$$

with

$$
\begin{aligned}
\dot{f}_{l}^{\prime \prime}\left(\lambda, \lambda^{\prime}\right) & =\left.\frac{\partial}{\partial q^{2}}\left\langle\dot{f}_{l}\left(\lambda \mathbf{q}, \lambda^{\prime} \hat{\mathbf{q}}^{\prime}\right)\right\rangle_{\hat{\mathbf{q}}^{\prime}}\right|_{q^{2}=0}=\frac{-\lambda^{2}\left(1+\lambda^{\prime}\right)^{2}+\lambda^{2}\left(3+2 \lambda^{\prime}\right) r_{l}}{12 \lambda^{\prime}\left(1+r_{l}\right)\left[\left(1+\lambda^{\prime}\right)^{2}+r_{l}\right]^{2}}, \\
\dot{\varphi}_{l}^{\prime \prime}(\lambda) & =\left.\dot{\chi}_{l}(0) \frac{\partial}{\partial q^{2}}\left\langle\left|\hat{\mathbf{q}}^{\prime}+\lambda \mathbf{q}\right|\right\rangle_{\hat{\mathbf{q}}^{\prime}}\right|_{q^{2}=0}=\frac{\lambda^{2}}{3\left(1+r_{l}\right)^{2}}, \\
\dot{\chi}_{l}^{\prime \prime}\left(\lambda, \lambda^{\prime}\right) & =\left.\frac{2}{1+r_{l}} \frac{\partial}{\partial q^{2}}\left\langle\tilde{G}_{l}\left(\lambda \mathbf{q}+\lambda^{\prime} \hat{\mathbf{q}}^{\prime \prime}+\hat{\mathbf{q}}^{\prime}\right)\right\rangle_{\hat{\mathbf{q}}^{\prime}, \hat{\mathbf{q}}^{\prime \prime}}\right|_{q^{2}=0}=\frac{\lambda^{2}\left[r_{l}-\left(1+\lambda^{\prime}\right)^{2}\right]}{6\left(1+r_{l}\right) \lambda^{\prime}\left[\left(1+\lambda^{\prime}\right)^{2}+r_{l}\right]^{2}}, \\
\dot{\alpha}_{l}\left(\lambda, \lambda^{\prime}\right) & =\left.\frac{1}{1+r_{l}} \frac{\partial}{\partial q^{2}}\left\langle\tilde{G}_{l}\left(\lambda \mathbf{q}+\lambda^{\prime} \hat{\mathbf{q}}^{\prime \prime}+\hat{\mathbf{q}}^{\prime}\right)\left|\lambda \mathbf{q}+\lambda^{\prime} \hat{\mathbf{q}}^{\prime \prime}\right|\right\rangle_{\hat{\mathbf{q}}^{\prime}, \hat{\mathbf{q}}^{\prime \prime}}\right|_{q^{2}=0}=\frac{\lambda^{2}\left[\left(1+\lambda^{\prime}\right)^{2}\left(2+\lambda^{\prime}\right)+\left(2+3 \lambda^{\prime}\right) r_{l}\right]}{12\left(1+r_{l}\right) \lambda^{\prime}\left[\left(1+\lambda^{\prime}\right)^{2}+r_{l}\right]} .
\end{aligned}
$$

To calculate the contributions of the BCS, ZS and $\mathrm{ZS}^{\prime}$ channel to Eq. (6.52), further averages are required:

$$
\left.\frac{\partial}{\partial q^{2}}\left\langle\dot{g}_{l}\left(\lambda \mathbf{q}, \lambda \hat{\mathbf{q}}^{\prime}\right)\right\rangle_{\hat{\mathbf{q}}^{\prime}}\right|_{q^{2}=0}=\frac{-\lambda\left[\lambda(1+\lambda)^{2}(2+3 \lambda)+(-4+(-2+\lambda) \lambda) r_{l}+2\left((1+\lambda)^{2}+r_{l}\right)^{2} \ln \left(\frac{1+r_{l}}{(1+\lambda)^{2}+r_{l}}\right)\right]}{12\left(1+r_{l}\right)\left[(1+\lambda)^{2}+r_{l}\right]^{2}},
$$




$$
\begin{aligned}
& \left.\frac{\partial}{\partial q^{2}}\left\langle\dot{f}_{l}\left(\lambda \mathbf{q}, \lambda \hat{\mathbf{q}}^{\prime}\right)\left|\lambda \mathbf{q}+\lambda \hat{\mathbf{q}}^{\prime}\right|\right\rangle_{\hat{\mathbf{q}}^{\prime}}\right|_{q^{2}=0}=\frac{\lambda\left[8\left(1+r_{l}\right)+\lambda\left[(3+\lambda)^{2}(3+\lambda(2+\lambda))+2(10+\lambda(6+\lambda)) r_{l}+r_{l}^{2}\right]\right]}{24\left(1+r_{l}\right)\left[(1+\lambda)^{2}+r_{l}\right]^{2}} \\
& +\frac{2\left(1-\lambda^{2}+r_{l}\right)\left[(1+\lambda)^{2}+r_{l}\right]^{2} \operatorname{arctanh}\left(\frac{\lambda \sqrt{-r_{l}}}{1+\lambda+r_{l}}\right)+\left(1+\lambda^{2}+r_{l}\right)\left[(1+\lambda)^{2}+r_{l}\right]^{2} \sqrt{-r_{l}} \ln \left(\frac{1+r_{l}}{(1+\lambda)^{2}+r_{l}}\right)}{24\left(1+r_{l}\right)\left[(1+\lambda)^{2}+r_{l}\right]^{2} \sqrt{-r_{l}}}, \\
& \left.\frac{\partial}{\partial q^{2}}\left\langle\dot{h}_{l}\left(\lambda \mathbf{q}, \lambda \hat{\mathbf{q}}^{\prime}\right)\right\rangle_{\hat{\mathbf{q}}^{\prime}}\right|_{q^{2}=0}= \\
& -\lambda \frac{\left[(\lambda-1)(1+\lambda)^{2}\left(\lambda+\lambda^{2}+2 r_{l}\right)+\lambda r_{l}^{2}\right] \sqrt{-r_{l}}+\left(1-\lambda^{2}-r_{l}\right)\left[(1+\lambda)^{2}+r_{l}\right]^{2} \operatorname{arctanh}\left(\frac{\lambda \sqrt{-r_{l}}}{1+\lambda+r_{l}}\right)}{6\left(1+r_{l}\right)\left[(1+\lambda)^{2}+r_{l}\right]^{2} \sqrt{-r_{l}}}, \\
& \left.\dot{\chi}_{l}(0) \frac{\partial}{\partial q^{2}}\left\langle\left|\hat{\mathbf{q}}^{\prime \prime}+\lambda \mathbf{q}\right|\left|\hat{\mathbf{q}}^{\prime \prime}+\lambda \hat{\mathbf{q}}^{\prime}\right|\right\rangle_{\hat{\mathbf{q}}^{\prime}, \hat{\mathbf{q}}^{\prime \prime}}\right|_{q^{2}=0}=\frac{\lambda^{2}\left(3+\lambda^{2}\right)}{9\left(1+r_{l}\right)^{2}}, \\
& \left.\frac{\partial}{\partial q^{2}}\left\langle\dot{\chi}_{l}\left(\left|\lambda \hat{\mathbf{q}}^{\prime}+\lambda \mathbf{q}\right|\right)\left|\lambda \hat{\mathbf{q}}^{\prime}+\lambda \mathbf{q}\right|^{2}\right\rangle_{\hat{\mathbf{q}}^{\prime}}\right|_{q^{2}=0}= \\
& \frac{\lambda^{2}(1+\lambda)^{2}(4+3 \lambda)+\lambda^{2}(4+5 \lambda) r_{l}+\lambda\left[(1+\lambda)^{2}+r_{l}\right]^{2} \ln \left(\frac{(1+\lambda)^{2}+r_{l}}{1+r_{l}}\right)}{6\left(1+r_{l}\right)\left[(1+\lambda)^{2}+r_{l}\right]^{2}} .
\end{aligned}
$$

Using these expressions to calculate $\dot{\Gamma}_{\infty}^{(4)}$ from Eqs. 6.106.166.236.246.25) one can determine $\eta$ via Eq. 6.52). One can check that to linear order in $\lambda$ one has

$$
\frac{\partial}{\partial q^{2}}\left\langle\dot{\Gamma}_{l}^{(4)}\left(\lambda \mathbf{q}, \lambda \hat{\mathbf{q}}^{\prime}, \lambda \hat{\mathbf{q}}^{\prime}, \lambda \mathbf{q}\right)\right\rangle_{\hat{\mathbf{q}}^{\prime}}{ }_{q^{2}=0} \simeq \lambda\left(\partial_{l} a_{l}+\partial_{l} b_{l}\right) / 3+2 \lambda\left(a_{l}+b_{l}\right) \eta_{l} / 3
$$

which, after performing the $\lambda$-integration, reproduces at the fixed point the result Eq. (6.39).

[1] F. Dalfovo, S. Giorgini, L. P. Pitaevskii, and S. Stringari, Rev. Mod. Phys. 71, 463 (1999).

[2] J. O. Andersen, Rev. Mod. Phys. 76, 599 (2004).

[3] G. Baym, J.-P. Blaizot, M. Holzmann, F. Laloe, and D. Vautherin, Phys. Rev. Lett. 83, 1703 (1999).

[4] N. V. Prokofe'ev, O. Ruebenacker, and B. Svistunov, cond-mat/0401002

[5] G. Baym, J.-P. Blaizot, M. Holzmann, F. Laloe, and D. Vautherin, Eur. Phys. J. B 24, 107 (2001).

[6] C. Wetterich, Phys. Lett. B 301, 91 (1993).

[7] T. R. Morris, Int. J. Mod. Phys. A9, 2411 (1994).

[8] S. Ledowski, N. Hasselmann, and P. Kopietz, Phys. Rev. A 69 , 061601(R) (2004).

[9] M. Bijlsma and H. T. C. Stoof, Phys. Rev. A 54, 5085 (1996).

[10] J. O. Andersen and M. Strickland, Phys. Rev. A 60, 1442 (1999).

[11] F. Pistolesi, C. Castellani, C. Di Castro, and G. C. Strinati, Phys. Rev. B 69, 024513 (2004).

[12] P. Kopietz, Nucl. Phys. B 595, 493 (2001).

[13] P. Kopietz and T. Busche, Phys. Rev. B 64, 155101 (2001).

[14] P. Arnold and G. Moore, Phys. Rev. Lett. 87, 120401 (2001).
[15] V. A. Kashurnikov, N. V. Prokof'ev, and B. V. Svistunov, Phys. Rev. Lett. 87, 120402 (2001).

[16] H. Kleinert, Mod. Phys. Lett. B 17, 1011 (2003).

[17] B. Kastening, Phys. Rev. A 69 , 043613 (2004).

[18] R. Shankar, Rev. Mod. Phys. 66, 129 (1994).

[19] P. Arnold, G. Moore, and B. Tomásik, Phys. Rev. A 65, 013606 (2001).

[20] J. Polchinski, Nucl. Phys. B 231, 269 (1984).

[21] J. Zinn-Justin, Quantum Field Theory and Critical Phenomena, (Clarendon Press, Oxford, 1989).

[22] R. Guida and J. Zinn-Justin, J. Phys. A 31, 8103 (1998).

[23] M. Campostrini, M. Hasenbusch, A. Pelissetto, P. Rossi, and E. Vicari, Phys. Rev. B 63, 214503 (2001).

[24] S. Ledowski and P. Kopietz, J. Phys.: Condens. Matter 15, 4779 (2003).

[25] J. D. Reppy, B. C. Crooker, B. Hebral, A. D. Corwin, J. He, and G. M. Zassenhaus, Phys. Rev. Lett. 84, 2060 (2000).

[26] T. R. Morris, Nucl. Phys. B 458, 477 (1996).

[27] T. Busche, L. Bartosch, and P. Kopietz, J. Phys. C: Cond. Matt. 14, 8513 (2002).

[28] C. Wetterich, Int. J. Mod. Phys. A16, 1951 (2001). 